\title{
AICHA: An atlas of intrinsic connectivity
}

\section{of homotopic areas}

\author{
MarcJoliot $^{\mathrm{a}}$, Gaël Jobard ${ }^{\mathrm{a}}$, Mikaël Naveau ${ }^{\mathrm{a}}$, Nicolas Delcroix ${ }^{\mathrm{b}}$, Laurent Petit $^{\mathrm{a}}$, \\ Laure Zago ${ }^{a}$, Fabrice Crivello ${ }^{\mathrm{a}}$, Emmanuel Mellet ${ }^{\mathrm{a}}$, Bernard Mazoyer $^{\mathrm{a}}$, \\ NathalieTzourio-Mazoyer ${ }^{\mathrm{a}}$ \\ a GIN, UMR 5296, CNRS, CEA, Bordeaux University, Bordeaux, France \\ ${ }^{\mathrm{b}}$ GIP CYCERON, UMS 3408, Caen F-14000, France
}

\begin{abstract}
Atlases of brain anatomical ROIs are widely used for functional MRI data analysis. Recently, it was proposed that an atlas of ROIs derived from a functional brain parcellation could be advantageous, in particular for understanding how different regions share information.

However, functional atlases so far proposed do not account for a crucial aspect of cerebral organization, namely homotopy, i.e. that each region in one hemisphere has a homologue in the other hemisphere.
\end{abstract}

We present AICHA (for Atlas of Intrinsic Connectivity of Homotopic Areas), a functional brain ROIs atlas based on resting-state fMRI data acquired in 281 individuals. AICHA ROIs cover the whole cerebrum, each having 1 - homogeneity of its constituting voxels intrinsic activity, and 2-a unique homotopic contralateral counterpart with which it has maximal intrinsic connectivity. AICHA was built in 4 steps: (1) estimation of resting-state networks (RSNs) using individual resting-state fMRI independent components, (2) $k$-means clustering of voxel-wise group level profiles of connectivity, (3) homotopic regional grouping based on maximal inter-hemispheric functional correlation, and (4) ROI labeling.

AICHA includes 192 homotopic region pairs (122 gyral, 50 sulcal, and 20 gray nuclei). As an application, we report inter-hemispheric (homotopic and heterotopic) and intra-hemispheric connectivity patterns at different sparsities.

ROI functional homogeneity was higher for AICHA than for anatomical ROI atlases, but slightly lower than for another functional ROI atlas not accounting for homotopy.

AICHA is ideally suited for intrinsic/effective connectivity analyses, as well as for investigating brain hemispheric specialization. 


\section{Introduction}

Over the previous ten years, the use of brain parcellation atlases (Evans et al., 2012) has become more frequent with the introduction of resting state functional magnetic resonance imaging (fMRI) connectivity analysis (Biswal et al., 1995). Within this framework, atlas based sets of regions of interest (ROI) are used to compute a brain graph, i.e., a model of the human brain functional connectome (Bullmore and Bassett, 2011). As shown by Craddock et al. (2012), region-based analysis has advantages compared with voxel-based analysis, including better sensitivity, interpretability and computational time. Furthermore, the reduction of the dimensionality of the fMRI data makes the problem statistically manageable by lowering the required number of statistical tests (Zalesky et al., 2012).

Today, several atlases are available, and each atlas has specific properties that should be considered with respect to the user's needs. The available atlases for functional/intrinsic connectivity and graph analyses can be characterized by the dataset of images that served in their elaboration, the target space that had been chosen for normalization, the neuroanatomo/functional parameters that drive the parcellation, and the parcellation algorithm.

The datasets involved in atlas creation define their "specific versus generic" nature. At one end are the most "specific" atlases, that are built from a single-subject dataset (such as in the AAL case (Tzourio-Mazoyer et al., 2002); at the other end are the most "generic" atlases, that are built from datasets representative of the general population. Note that elaboration of a truly "generic" atlas is out of reach, since it would request datasets for all ages, ethnicities, etc. Rather, available generic atlases are based on samples representative of some specific population, balancing for some given phenotypes, such as sex (Craddock et al., 2012), or conversely, selecting a specific phenotype, such right-handedness (Shen et al., 2013).

Regarding the target space, two main choices are available, namely volume (Ashburner and Friston, 2005) or surface (Dale et al., 1999). While the surface space has been demonstrated to be more accurate (Jo et al., 2007), the volume space is important when one is interested in tissue other than the cortical gray matter mantle, such as white matter or nuclei. The partial or complete parcellation proposed for a given brain tissue class should also be considered in atlas selection. For example, network centrality graph analysis (Bullmore and Bassett, 2011) can be critically affected in the case of partial coverage atlases because such analyses consider the strength of the connection between all graph regions. For example, centrality analysis based on an incomplete parcellation atlas or a complete cortex coverage atlas can lead to discrepant sets of "hub" region identification.

For each atlas, there are some neuro-anatomo-functional parameters that guide the parcellation scheme and constrain the definition of landmarks that constitute the boundaries of the atlas regions. Three types of landmarks are used for gray matter parcellation, including sulci (anatomical landmarks, Tzourio-Mazoyer et al., 2002, Kennedy et al., 1998), borders of cytoarchitectonic areas (Caspers et al., 2006) or limits defined from functional properties, such as the borders of spatially coherent regions of homogeneous functional connectivity (Craddock et al., 2012). Note that the homogeneity in the regions of interest of a given atlas will depend on the parameters that guide its parcellation scheme.

The atlas building constraints that are explicitly or implicitly associated with the parcellation scheme, such as the choice of the landmarks, has a strong impact on the topology of the 
parcellation in terms of the shape and number of parcels and thus on the atlas' final resolution.

The aim of the present work was to design an atlas suitable for functional/intrinsic connectivity and graph analysis that would consider and benefit from a major characteristic of brain organization, namely homotopy. Homotopy corresponds to the fact that the two hemispheres have comparable organization regarding macroscopic anatomy, cytoarchitecture and large-scale functional organization (Fuster, 1998, Mesulam, 1990) and are for the most part connected to each other by the white matter tracts of the corpus callosum, which originate from and terminate in the 4th cortical layer. As a consequence, almost all areas from identical cortical structures and with identical hierarchical levels are connected through the corpus callosum in a mirroring way. However, this cortical symmetry is not perfect because of the global torsion of the brain, the Yaklovian torque, which makes difficult a point-to-point correspondence between cortical areas that are functionally homotopic (Toga and Thompson, 2003). In addition, the torque goes along with asymmetries in sulcus depth and position in relation to differences in asymmetries in neighboring cortices (Lyttelton et al., 2009); the largest sulcal and cortical asymmetries in newborns and adults are located at the termination of the Sylvian fissure and at the superior temporal sulcus (Hill et al., 2010). These gross morphological differences across hemispheres increase the difficulty in defining homotopic regions in the temporal and inferior parietal areas. For example, in order to calculate regional asymmetries, one must define what is meant by homotopic regions. This definition can be based either on anatomical atlases, such as AAL (Tzourio-Mazoyer et al., 2002), which use a rough spatial metric based on the position of the regions relative to the sulci, or on a cytoarchitectonic atlas based on both anatomical symmetry in position and differences in the cortical lamination patterns (Caspers et al., 2006). In functional atlases, criteria based on the functional characteristics of the regions of the atlas areas have not yet been proposed to define homotopic areas. However, it is common to observe homotopic-like patterns in resting data analysis using seed based analysis (van den Heuvel and Hulshoff Pol, 2010, Jo et al., 2012) or network decomposition methods (Beckmann et al., 2005, Naveau et al., 2012, Yeo et al., 2011). Furthermore, Stark et al. (2008) have reported that the highest mean functional connectivity is observed between inter-hemispheric symmetrical areas compared with other inter-hemispheric or intra-hemispheric connections. These observations support that homotopic organization is a fundamental feature of the functional organization of the cortex and plead for the definition of homotopic areas based on functional, rather than anatomical, criteria.

The Atlas of Intrinsic Connectivity of Homotopic Areas (AICHA) we propose here is thus a population-level, cerebrum gray matter brain atlas of homotopic regions based on a time correlation structure of the resting brain. We designed AICHA based on the following properties: 1 -a large dataset of 281 healthy participants balanced for handedness and gender; 2 - volumetric MNI space of normalization to cover the gray matter of the whole cerebrum, including the sub-cortical gray nuclei; 3 - time correlation structure of the functional resting state signal (intrinsic connectivity) as the basis for landmark definition; and 4-choice of an algorithm that permits a homotopic parcellation to maximize the weight of the functional signal at the final step. We describe the AICHA atlas building, its dataset regional functional homogeneity (i.e., the intra-regional similarity level of the voxel blood oxygen level dependent time-series), compare it to four existing atlases, and provide the intra- and interhemispheric intrinsic connectivity patterns at different thresholds of regional connection strength. 


\section{Materials and methods}

\subsection{Participants}

Two hundred eighty-one healthy adults (137 women, 144 men) aged 18-57 years (25 \pm 6 years, mean $\pm \mathrm{SD}$ ) were included in this study. Subject handedness was self-reported, and their manual preference strength was measured using the Edinburgh score (ES, mean $\pm \sigma$, (Oldfield, 1971). 133 subjects were right-handed (66 women, ES = $94 \pm 10 ; 67$ men, ES = 91 \pm 13 ) and 148 subjects were left-handed ( 71 women, $\mathrm{ES}=-63 \pm 39 ; 77 \mathrm{men}, \mathrm{ES}=-63 \pm 41$ ). All subjects provided informed written consent, and the local ethics committee (CPP de Basse-Normandie, France) approved the study.

\subsection{Data acquisition and pre-processing}

\subsubsection{Imaging methods}

Imaging was performed on a 3 Tesla MRI scanner (Achieva Philips, Best, The Netherlands). Spontaneous brain activity was monitored using blood oxygen level dependent (BOLD) fMRI while the participants performed an 8 -min resting state condition $(\mathrm{T} 2 *$-EPI, sequence parameters: 240 volumes; $\mathrm{TR}=2 \mathrm{~s}$; $\mathrm{TE}=35 \mathrm{~ms}$; flip angle $=80^{\circ} ; 31$ axial slices; and $3.75 \times$ $3.75 \times 3.75 \mathrm{~mm} 3$ isotropic voxel size). Immediately preceding the fMRI scan, the subjects were instructed to "keep their eyes closed, relax, refrain from moving, stay awake and let their thoughts come and go". Prior to the fMRI session, high-resolution 3D T1-weighted structural MR brain images (sequence parameters: $\mathrm{TR}=20 \mathrm{~ms}$; $\mathrm{TE}=4.6 \mathrm{~ms}$; flip angle $=10^{\circ}$; inversion time $=800 \mathrm{~ms}$; turbo field echo factor $=65$; sense factor $=2$; field of view $=256 \times 256 \times 180$ $\mathrm{mm} 3$; and $1 \times 1 \times 1 \mathrm{~mm} 3$ isotropic voxel size) and T2*-weighted multi-slice images (T2*weighted fast field echo [T2*-FFE], sequence parameters: TR $=3.5 \mathrm{~ms}$; TE $=35 \mathrm{~ms}$; flip angle $=90^{\circ}$; sense factor $=2 ; 70$ axial slices; and $2 \times 2 \times 2 \mathrm{~mm} 3$ isotropic voxel size) were acquired. The subjects did not perform cognitive training or any other task prior to image acquisition.

\subsubsection{Data pre-processing}

Pre-processing was performed using Statistical Parametric Mapping subroutines (SPM5, Wellcome Department of Neurology, London, UK; www.fil.ion.ucl.ac.uk/spm) completed by in-house MATLAB-based software. Each subject's anatomical T1-weighted volume was segmented into three brain tissue classes (gray matter, GM; white matter, WM; and cerebrospinal fluid, CSF) and spatially normalized using specific cerebral tissue templates built from the T1-weighted images of 80 subjects ( 40 men) acquired with the same scanner and acquisition parameters (Template resolution of $2 \times 2 \times 2 \mathrm{~mm} 3$ voxels; bounding box, $\mathrm{x}=$ -90 to $90 \mathrm{~mm}, \mathrm{y}=-126$ to $91 \mathrm{~mm}, \mathrm{z}=-72$ to $109 \mathrm{~mm}$ ) and normalized to the stereotaxic space of the Montreal Neurological Institute (MNI) template (Ashburner and Friston, 2005). The fMRI data were corrected for slice timing differences and motions ( 6 parameters: 3 translations and 3 rotations) and registered to the T2*-FFE volume. The fMRI data were then spatially normalized combining the $\mathrm{T} 2 *$-FFE to $\mathrm{T} 1$-weighted registration matrix and the $\mathrm{T} 1-$ weighted stereotaxic normalization matrix and spatially smoothed (Gaussian $6 \mathrm{~mm}$ full width at half maximum filter). Finally, the time series for WM, CSF (average time series of voxels that belong to each tissue class), the six motion parameters and the temporal linear trend were regressed out of the fMRI data.

\subsection{Atlas building methodology}

The methodology is subdivided in 4 stages that are summarized on the flow chart in Fig. 1, with intermediate results shown in Fig. 2. The first stage covers the estimation of resting-state networks (RSN's) using an algorithm of classification of individually extracted independent 
components. The second stage covers the making of a template of regions of interest (ROI's) based on these RSN's using a k-means clustering algorithm. The rationale for choosing such a RSN-ROI based (rather than voxel-level) approach was three-fold: 1 - the need for reducing spatially-extended noise-related artifacts in rs-fMRI correlation maps (Salimi-Khorshidi et al., 2014), 2-use RSN decomposition to capture the group reproducible functional correlation patterns 3-the fact that most RSN's are spatially symmetrical, a feature we considered as a strong advantage in the context of building an atlas of homotopic ROI's. The third stage concerns the homotopic regional grouping procedure and the parcellation refinement, while the fourth stage deals with the atlas labeling procedure.

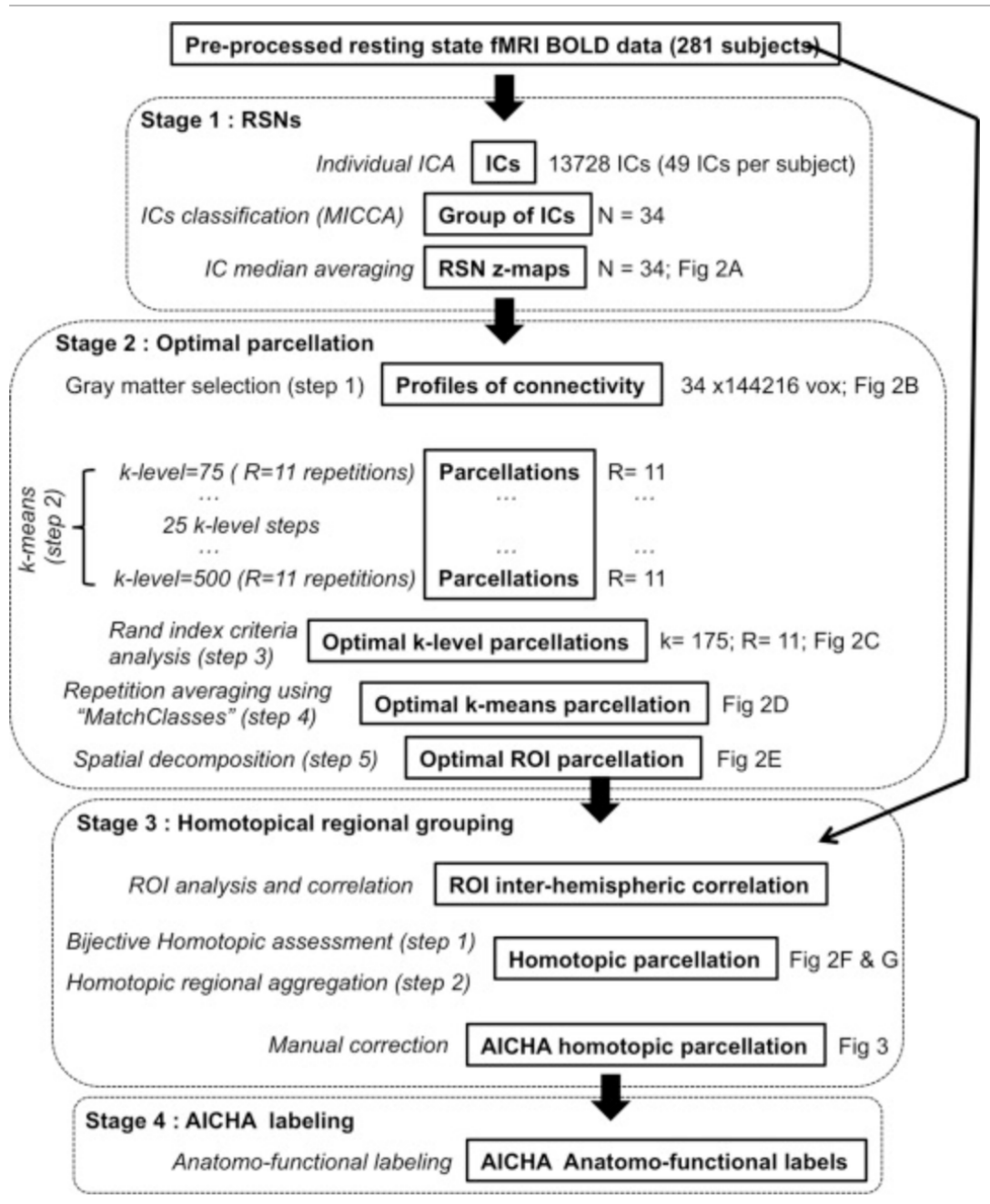

Download : Download full-size image

Fig. 1. Flow chart of the atlas construction. The stages and steps have been described in the text. Each line lists the data computed (square box and bold); on the left, the method used (italic) is shown and on the right, Supplementary information is shown and in some cases, there is a reference to the data in Fig. 2. RSN: Resting state network; IC: Independent component; ICA: Independent component analysis; MICCA: Multi-scale clustering of individual component algorithm; ROI: Region of interest; $N$ : number of RSN; $R$ : number of $k$-means repetition; $k$ or $k$-level: Number of parcels of the $k$-means algorithm. 


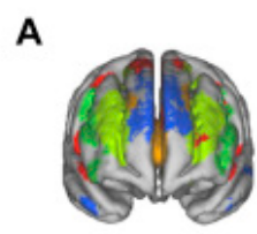

Frontal (5)

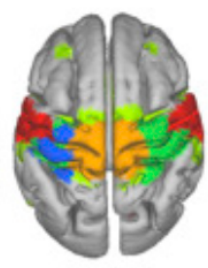

SM1 (5)

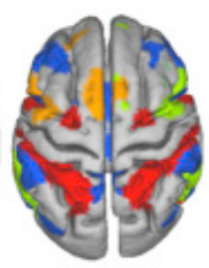

FPT1 (5)

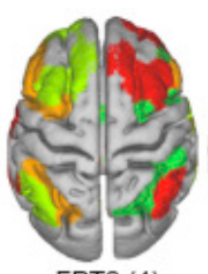

FPT2 (4)

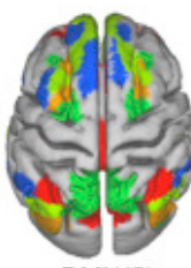

DMN(5)

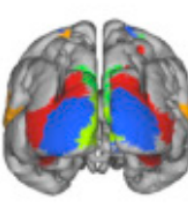

$\mathrm{V}-\mathrm{A}(5)$

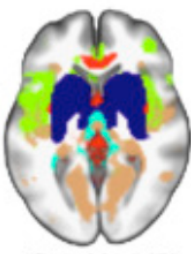

Sub-Med (5)
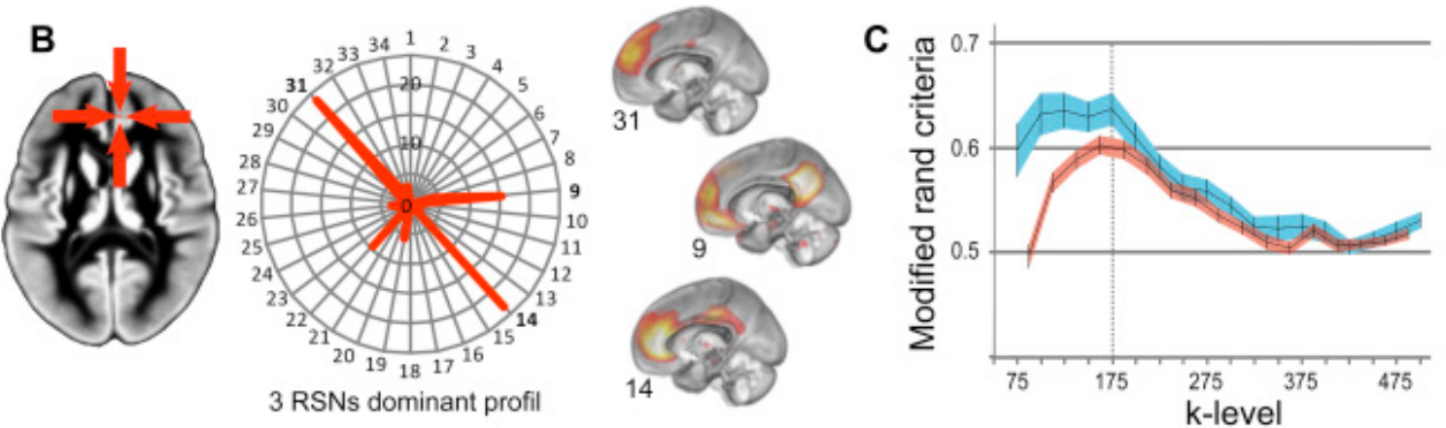

3 RSNs dominant profil

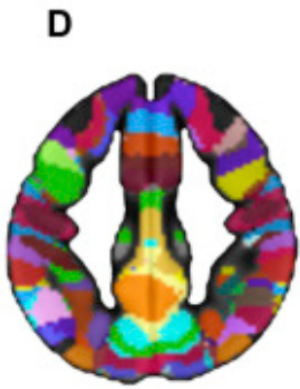

k-means $n=175$
E

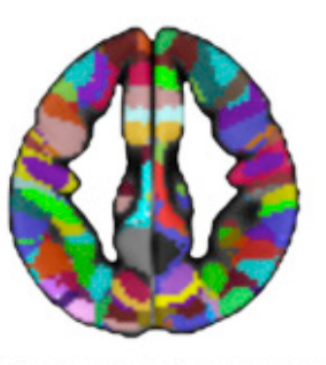

$\mathbf{F}$

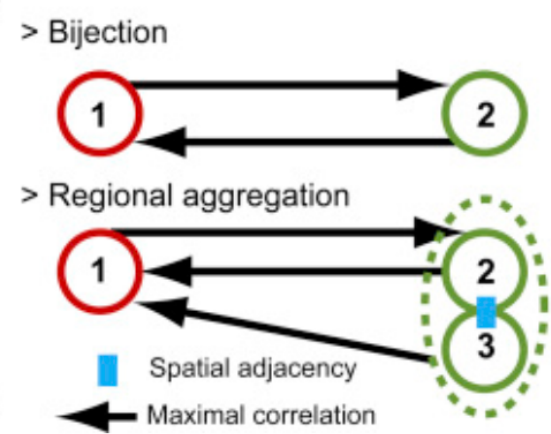

G

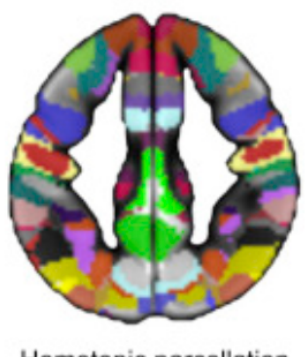

Download : Download full-size image

Fig. 2. Major steps of the atlas construction. (A) A 3-dimensional or 2-D rendering of the 34 resting state networks identified by MICCA (SM: Sensory-motor; FPT: Fronto-parietotemporal; DMN: Default Mode Network; V-A: Visual-Auditory; Sub-Med: Subcortical and medial). (B) Illustration of the connectivity profile of one voxel. (C) Selection of the optimal parcellation using the Rand index criteria corrected for agreement by chance. The blue shade curve shows the rand criteria and confidence interval computed between the 11 repetitions of the $k$-level. The red shade curve shows the same values between the repetitions of each successive $k$-level. The dashed vertical bar indicates the optimal $k$-level value that precedes the intersection of the 2 confidence intervals. (D) Optimal parcellation. (E) Optimal parcellation after hemispheric separation. (F) Rules for the homotopic regional determination and grouping. (G) Homotopic parcellation (the same color is used for homotopic regions). (For interpretation of the references to color in this figure legend, the reader is referred to the web version of this article.)

\subsubsection{Estimation of resting-state networks (Stage 1)}


We used a multi-scale clustering of individual components (MICCA) to estimate group-level resting-state networks (RSNs). This algorithm has been fully described elsewhere (Naveau et al., 2012); thus, we only present the key features of the procedure. First, we computed individual independent components (ICs) for each subject using probabilistic independent component analysis provided by the FMRIB Software Library (FSL,

http://www.fMRIb.ox.ac.uk/fsl) (Smith et al., 2004) under the name MELODIC (Multivariate exploratory linear optimized decomposition into independent components, version 3.10). The number of ICs was estimated for each subject (i.e., model order $=48 \pm 6$, mean $\pm \mathrm{SD}, \mathrm{N}=$ 281) using Laplace approximation (Minka, 2000). Each extracted component was described as a spatial z-map (zIC) built to quantify each voxel membership to the specific IC. These zvalues represent the correlation between the voxel time series and IC time series divided by the standard error of the residual noise (Beckmann et al., 2005). In a second step, the MICCA method clustered zICs across individuals according to their spatial similarity and provided a group of zICs (gICs) that corresponded to the same signal source. These two steps were replicated 20 times because we used a random (stochastic) algorithm for ICA. This algorithm relies on a pseudo-random initialization and, consequently, two decompositions of the same data led to two different solutions. The results were aggregated using Icasso (Himberg et al., 2004), and we retained group-level components repeatedly identified in more than half of the repetitions $(>10)$. Forty-five gICs were identified using this procedure. The gICS that demonstrated a maximal proportion of cerebrum gray matter $(\mathrm{N}=34)$ were considered RSNs (Naveau et al., 2012). The 11 discarded gICS were primarily localized in the cerebrospinal fluid (6), the white matter (3) and the cerebellum or cerebral trunk (2). The 34 selected RSNs covered $98 \%$ of the cerebrum gray matter.

Using the individual zICs that belong to each RSN, we created a median z-map (zICmed) that coded how strongly the voxels belonged to the corresponding RSN. Thus, the median of the z-value distribution across individual zIC maps included in the considered gICs was assigned to each voxel of zICmed. For display purposes, a thresholded map (gamma-Gaussian mixture model $p>0.95$ ) of each RSN was computed, both 3D and 3 orthogonal slice renderings are presented in Fig. 2A and Supplementary Fig. S1, respectively.

\subsubsection{Construction of the optimal parcellation (Stage 2)}

The construction of the optimal parcellation was decomposed in 5 steps.

\subsubsection{Step 1: Profiles of connectivity}

Using the ICmed z-maps, we defined voxel-wise profiles of connectivity across the RSNs. We retained voxels of the sample average gray matter probability map that indicated a gray matter probability above $25 \%$. The cerebellum and brain stem were subsequently removed using a ROI manually defined on the average T1-weighted volume (144,216 voxels selected). For each selected voxel (v), a connectivity profile was created as an N-dimensional vector, such as $\mathrm{Cp}(\mathrm{v})=[\mathrm{zICmed}, 1(\mathrm{v})$, zICmed,2(v),.., $\mathrm{zICmedN}(\mathrm{v})]$, where $\mathrm{N}$ is the number of resting-state networks (here $\mathrm{N}=34$ ). Each element of the "connectivity profile" is thus a statistical measure of its membership to the corresponding network. The z-values of connectivity were normalized so that $\sum \mathrm{Cp}(\mathrm{v})=1$ (the negative values were set to zero prior to the normalization) (Fig. 2B). Each brain voxel was thus attributed a normalized connectivity profile along the $\mathrm{N}=34$ dimensions.

\subsubsection{Step 2: k-means parcellation}

The Matlab-based k-means algorithm (provided by the Statistical Toolbox) was chosen as the parcellation algorithm. The whole set of connectivity profiles, $\mathrm{Cp}(\mathrm{v})$ (v belonging to the gray 
matter, see above), was submitted to multiple k-mean decompositions with $18 \mathrm{k}$-level initial number of clusters that ranged from 75 to 500 in steps of 25 . For each k-level of partition, 11 repetitions using random seed initialization were computed, which led to 11 parcellations. In the k-means clustering, the squared Euclidean distance was used as a measure of dissimilarity between average cluster connectivity profiles.

\subsubsection{Step 3: Definition of the optimal k-level used in the parcellation}

To define the optimal k-level, the resulting partitions were compared using the Rand index criteria corrected for agreement by chance (Hubert and Arabie, 1985). Two voxels are in agreement in partition $\mathrm{P} 1$ and $\mathrm{P} 2$ if they are placed in the same cluster in P1 and in the same cluster in $\mathrm{P} 2$ or if they are in different clusters in P1 and P2. The rand index corresponds to the proportion of voxel pairs in agreement. Because the Rand index is not null in the case of random parcellation (Hubert and Arabie, 1985), we used a Rand index "corrected for chance" using a generalized hypergeometric distribution null model. Two corrected-Rand indexes were computed: one index between each repetition of a k-level (intra-level agreement) and one index between repetitions of each successive k-level (inter-level agreement). Confidence intervals for both intra and inter k-level corrected-Rand indexes were then computed. We observed that the intra-level agreement (Fig. 2C, blue curve) attained a high-level plateau, i.e., a maximal stability, in the 100 and $175 \mathrm{k}$-level range. The optimal regional parcellation was set at the highest level of partition in the maximum intra-level agreement range $(\mathrm{k}-\mathrm{level}=$ 175). The inter-level agreement (Fig. 2C, red curve) was below the intra-level agreement up to the k-level of 175 and was at the same level as the intra-level agreement beyond this value. These findings indicate that at higher levels, the difference between two successive parcellations was in the same range as the difference measured by the replication. Thus, there was no clear benefit in increasing the subdivision. The use of this criterion resulted in a parcellation of the 144,216 voxels in 175 clusters.

\subsubsection{Step 4: Construction of the optimal parcellation}

Once the optimal k-level was determined, the 11 parcellations of the $175 \mathrm{k}$-level solutions were collapsed into a single parcellation. We used a two-step procedure (Fig. 2D). In the first step, we maximized the matching of the class denomination between one randomly chosen parcellation and the 10 other parcellations. For each pair, we used the "matchClasses" function of the "e1071" R-package (Meyer et al., 2012). For each pair, we built a contingency matrix that represented the number of voxels that belonged to each possible pair of parcels between the 2 considered parcellations. Using permutation of the rows and columns of this matrix, the algorithm maximizes the trace of the matrix (i.e., the sum of the diagonal values) (Parameters of "matchClasses": method ="exact", maxexact $=9$, iteration for greedy search = 105). In the second step, each voxel was attributed to one parcel based on a maximum voting criteria of the 11 repetitions. Note, that for voxels with a matching vote, they were affected to the parcel they were connected to (edge connectivity criteria) and pseudo-randomly to one parcel for those that connected the two parcels.

\subsubsection{Step 5: Spatial decomposition of the parcellation}

Hemispheres were disconnected by removing the voxels that overlapped the inter-hemispheric fissure. The fissure geometry was defined as the average of the anatomical T1-weighted volumes (Supplementary Fig. S2). Following this virtual hemisphere disconnection, each cluster showing on both hemispheres appears as at least 2 spatially disconnected regions. In fact it could be more than two regions per cluster, as the k-means algorithm does not impose to get only one region because it does not consider the spatial information. To get the parcellation we performed a connected-component analysis using a voxel edge-connectivity 
heuristic. This heuristic states that one voxel is connected to neighbor voxels if they share a common edge, thus one voxel is connected to 18 neighbors. This step led to a parcellation in 221 and 239 regions in the left and right hemispheres, respectively (Fig. 2E).

\subsubsection{Homotopic regional grouping (Stage 3)}

We computed the average BOLD time-series for each region of the atlas in each subject. For each region identified, we identified the contralateral region that had the maximal average temporal Pearson correlation coefficient across subjects. Thus, we considered only the interhemispheric connections. The homotopic relationship was defined using two steps (Fig. 2F):

\subsubsection{Step 1}

Two regions A and B were labeled as "homotopic" if they fulfilled the following three conditions: region A shows a maximal temporal correlation with region B; region B shows a maximal temporal correlation with region $\mathrm{A}$; and no other region had a maximum correlation with A or B.

Following the first step, the parcellation comprised 460 regions, including 274 homotopic regions (60\% of the regions, 137 pairs) and 84/102 left/right orphans regions, i.e., regions that did not belong to a homotopic pair.

\subsubsection{Step 2}

If two spatially adjacent regions $\mathrm{B}$ and $\mathrm{C}$ show a maximal correlation with the contralateral region $\mathrm{A}$ and if region $\mathrm{A}$ shows a maximal temporal correlation with $\mathrm{B}$ or $\mathrm{C}$, then we merged $\mathrm{B}$ and $\mathrm{C}$ and labeled the regions (A; B U C) as "homotopic". Note that the resultant regions had to fulfill the Step 1 criteria.

After this step, the parcellation comprised 386 regions, including 360 homotopic regions (93\% of the regions, 180 pairs) and 26 (13 per hemisphere) orphan regions, i.e., regions that did not belong to a homotopic pair (Fig. 2G).

A visual inspection of the orphan regions revealed that they appeared as two non-neighboring regions in one hemisphere that had a maximal correlation with the same regions in the other hemisphere. In such cases, the target region generally consists of two foci linked by a thin continuum of voxels located either at the gray/white matter or gray/CSF interface. These continuums were manually removed, which created the same number of regions in each hemisphere. The homotopic regional grouping procedure was re-applied for identifying the homotopic couples. At the end, only 4 regions remained orphans: two regions in the frontal lobe and two regions in the parietal lobe (Supplementary Fig. S3). An analysis of the correlation pattern demonstrated that if the left and right parietal regions were maximally correlated, the right frontal region was maximally correlated with the left parietal region, although not significantly more than with the left frontal region (paired t-test correlation difference, $\mathrm{t}(280)=0.92, \mathrm{p}=0.36$ ). Consequently, we re-affected both frontal regions and both parietal regions as homotopic couples. The resulting final parcellation, which is referred to as the Atlas of Inter-Connected Homotopic Area (AICHA), is shown in Fig. 3. 

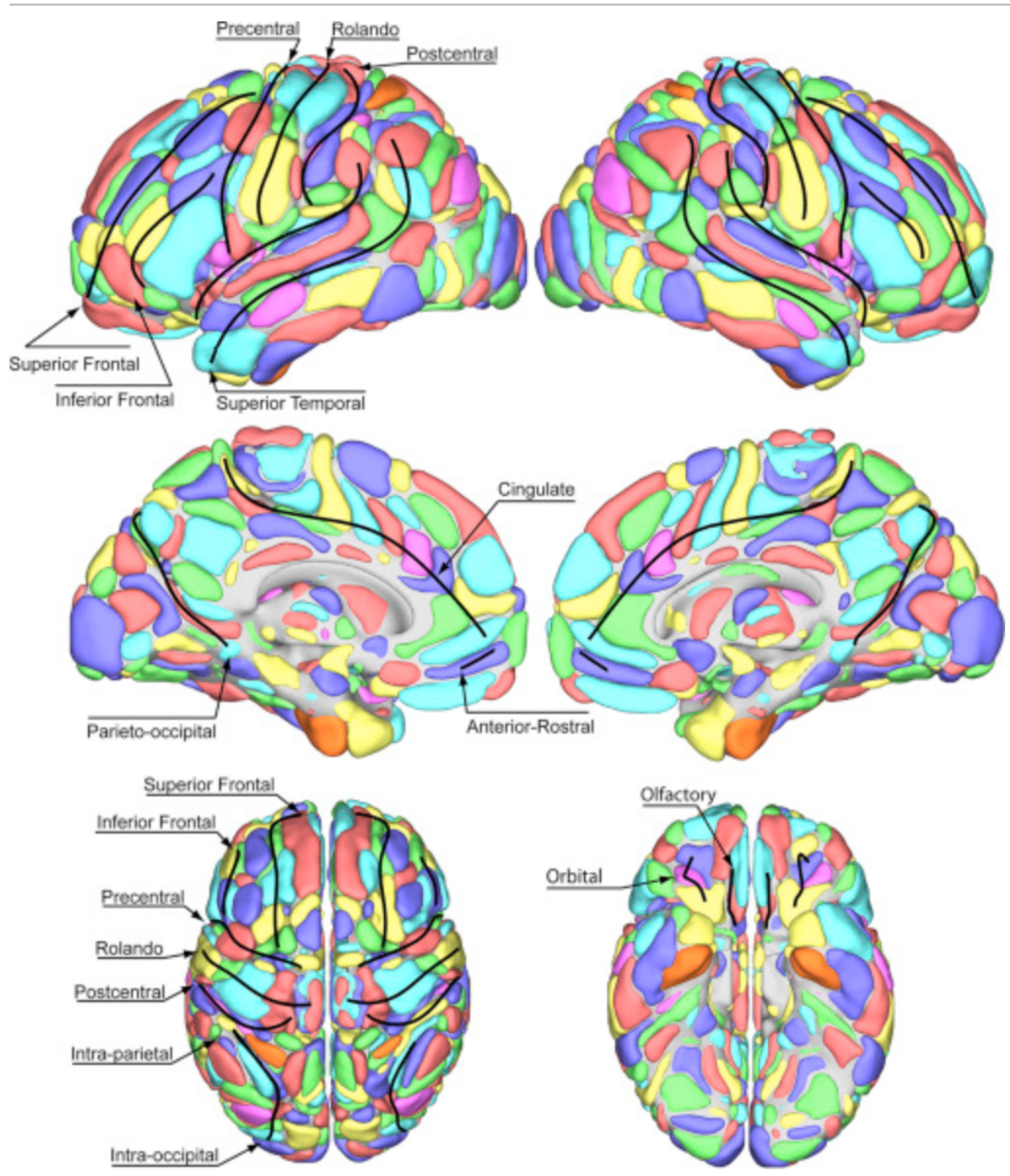

Download : Download full-size image

Fig. 3. Rendering of AICHA and the sulci used in the anatomical labeling.

\subsubsection{AICHA labeling (Stage 4)}

The labeling of the regions constituting AICHA was based on sulcal and gyral anatomical labels. The sulci were defined at the intersection of the T1-weighted structural MR average image convex hull and the CSF compartment (Joliot and Mazoyer, 1991) (Supplementary Fig. S4). The convex hull was computed using Caret software (Van Essen et al., 2001). The sulci and gyri were labeled using the rule used in the construction of the AAL atlas (TzourioMazoyer et al., 2002). AICHA parcellation encompasses both gyral regions located in the crown of the gyri and sulcal regions located in the depth of the sulci. With the addition of gray nuclei, 55 anatomical labels were defined in each hemisphere.

The anatomical scale was not sufficiently precise to describe the fine spatial resolution of AICHA; thus, additional labeling procedures were defined inside each anatomically labeled region. The different sub-regions were labeled using numbers that were attributed according 
to cumulative criteria: first from anterior to posterior, then from ventral to dorsal and finally from lateral to medial.

\subsection{Homotopic ROI pair volume asymmetry}

The normalized volume asymmetry (NVA) of each pair of homotopic regions was computed as the left minus right region volume difference divided by the mean volume of the pair of regions. Positive (or negative) values indicate that the left (or right) region has a larger volume than its contralateral region. The NVAs were submitted to an outlier analysis and we report the regions that exhibit NVA values below the 25 th and above the 75 th percentiles of the distribution.

\subsection{Regional functional homotopic correlation}

For each of the 281 participants and each region of AICHA, the mean BOLD fMRI timeseries was computed by averaging the signals of all voxels belonging to this region. Then, for each participant and each homotopic pair of regions, we computed the Pearson's linear correlation coefficient ( $\mathrm{r}$ ) between the region's BOLD fMRI time-series. Based on these individual results, the regional group-average homotopic correlations (AHC) and variation coefficient homotopic correlation (VHC) maps were computed. Note that the VHC map was computed at the basis of the z-transform of the homotopic correlation, taking into account the saturation effect of the correlation measures.

\subsection{Comparison of atlases}

We defined a regional functional homogeneity measure as the average Pearson correlation coefficient between the BOLD time series of all pairs of voxels belonging to a considered region. The regional functional homogeneity of AICHA was computed using an additional distinct resting state dataset ( 88 subjects), which was acquired and processed in the same manner as the AICHA generation dataset. The dataset was balanced for both gender and handedness ( 22 subjects per combination). These measures were compared with the measures obtained in the regions defined by 4 previously published and available atlases. Note that only the cerebrum gray matter regions were retained for this analysis. These atlases included the Automated Anatomical Labeling Atlas (referenced as AAL, 90 anatomical regions selected, Tzourio-Mazoyer et al., 2002), the anatomical based Harvard-Oxford Atlas (referenced here as Ha-Ox, 110 anatomical regions selected, Desikan et al., 2006), the Juelich cytoarchitectonic atlas (referenced here as Cyt-Jue, 90 cytoarchitectonic regions selected with a partial coverage of the cerebrum gray matter, Caspers et al., 2006) and one of the resting state based functional atlases provided by Craddock et al. (2012) (referenced here as FuncCra, 394 functional regions selected).

\subsection{Description of the atlas' regional functional correlations}

We were interested in characterizing the between-subject pairwise regional correlations based on AICHA's regions. We describe the functional inter-hemispheric (homotopic and heterotopic) and intra-hemispheric (left and right) connectivity patterns of the creation atlas dataset at different thresholds of connection strength.

For each of the 281 individuals and each region of AICHA, an individual regional BOLD fMRI time-series was computed by averaging signals from all voxels belonging to this region. Then, for each individual and each pair of regions, we computed the Pearson's linear correlation coefficient between the pair of regional BOLD fMRI time-series. 
Region pair correlation values $(73,536)$ were analyzed at a sparsity $(\mathrm{St})$ threshold going from 50 to $2 \%$ (step of $2 \%$ ), i.e., only the St percentage of highest correlation values was retained for each subject. For each threshold, the pairs that had correlation values above the chosen threshold for at least $95 \%$ of the participants were selected and were classified into the 4 categories: homotopic, heterotopic, intra-hemispheric left and intra-hemispheric right. The intra-hemispheric correlations were further analyzed for the proportion of bilateral (present in both hemispheres) or unilateral (present in only one hemisphere) occurrences.

\section{Results}

\subsection{AICHA description}

\subsubsection{General description of AICHA ROIs}

AICHA includes 192 pairs of homotopic ROIs (Fig. 3). Of these ROIs, 122 ROIs anatomically belong to 37 gyri (Table 1), 50 ROIs overlap 14 sulci (Table 2) and 20 ROIs are localized within the 4 subcortical gray matter nuclei (Table 2). The complete labels are provided as Supplementary material (see Supplementary Fig. S5), together with the ROI volumes and mass center coordinates. The average volume of the AICHA ROIs is $2.1 \pm 2$ $\mathrm{cm} 3$ (mean \pm S.D.) with a range from 0.16 to $10.2 \mathrm{~cm} 3$. A three-dimensional rendering of each region is provided in Supplementary Fig. S5. 
Table 1. Gyrus names used in the anatomical labeling of AICHA and the number of functional subdivisions.

\section{Gyrus anatomical name}

G_Frontal_Sup

G_Frontal_Mid

G_Frontal_Inf_Tri

G_Frontal_Sup_Orb

G_Frontal_Mid_Orb

G_Frontal_Inf_Orb

G_Parietal_Sup

G_SupraMarginal

G_Angular

G_Parietal_Inf

G_Occipital_Pole

G_Occipital_Lat

G_Occipital_Sup

G_Occipital_Mid

G_Occipital_Inf

G_Insula-anterior

G_Insula-posterior

G_Rolandic_Oper

G_Temporal_Sup

G_Temporal_Mid

G_Temporal_Inf

G_Temporal_Pole_Sup

G_Temporal_Pole_Mid

G_Frontal_Sup_Medial

G_Frontal_Med_Orb

G_Subcallosal

G_Supp_Motor_Area

G_Cingulum_Ant

G_Cingulum_Mid

G_Cingulum_Post

G_Paracentral_Lobule

G_Precuneus

G_Cuneus

G_Lingual

G_Hippocampus

G_ParaHippocampal

G_Fusiform

\section{Number of subparts}

5

2

2

5

3

1

5

4

1

2

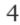

2

3

3

2

1

3

3

4

2

6

2

5

7 
Table 2. Sulcus and nucleus names used in the anatomical labeling of AICHA and the number of functional subdivisions.

$\begin{array}{lll}\text { Anatomical name } & \text { Sulcus/nucleus } & \text { Number of subparts } \\ \text { S_Superior_Frontal } & \text { S } & 6 \\ \text { S_Inferior_Frontal } & \text { S } & 2 \\ \text { S_Orbital } & \text { S } & 2 \\ \text { S_Olfactory } & \text { S } & 1 \\ \text { S_Precentral } & \text { S } & 6 \\ \text { S_Rolando } & \text { S } & 4 \\ \text { S_Postcentral } & \text { S } & 3 \\ \text { S_Intra-parietal } & \text { S } & 3 \\ \text { S_Intra-occipital } & \text { S } & 1 \\ \text { S_Superior_Temporal } & \text { S } & 5 \\ \text { S_Anterior_Rostral } & \text { S } & 1 \\ \text { S_Cingulate } & \text { S } & 7 \\ \text { S_Parieto-occipital } & \text { S } & 6 \\ \text { S_Calcarine } & \text { S } & 3 \\ \text { Amygdala } & \text { N } & 1 \\ \text { Caudate } & \text { N } & 7 \\ \text { Pallidum/Putamen } & \text { N } & 3 \\ \text { Thalamus } & \text { N } & \end{array}$

\subsubsection{Anatomical location of AICHA ROIs}

Overall, $74 \%$ of the ROIs (142 regions per hemisphere) were localized within a gyrus or a gray matter nucleus. These regions can thus be considered subparts of the anatomical regions based on sulcal landmarks. This was also the case for 18 sulcal-labeled regions per hemisphere that were primarily localized in one bank of a sulcus, including the precentral, postcentral, intra-parietal and parieto-occipital sulci ROIs (Table 2). The remaining sulcal regions (32 per hemisphere, $17 \%$ of the total) encompass both banks equally, and these ROIs represent "new" regions compared with the anatomically defined atlases. These regions include the superior and inferior frontal, orbital, anterior_rostral, olfactory, Rolando, intra_occipital, superior temporal, cingulate and calcarine sulcus ROIs (Table 2).

\subsubsection{Volume asymmetry of AICHA ROIs}

The normalized volume asymmetry (NVA) distribution of AICHA's ROIs was centered on zero showing no asymmetry. NVA absolute values larger than $61.8 \%$ (which corresponded to the 25th and 75th percentiles of the distribution) were observed in 8 leftward asymmetrical and 10 rightward asymmetrical ROIs (Table 3 ). 
Table 3. Homotopic region pair with normalized volumetric asymmetry (NVA) below the 25th and above the 75th percentiles of the distribution. NVA is defined as positive when the left regions are larger than their right homotopic counterparts. Left and right mass center MNI stereotaxic coordinates are also shown. Regions that exhibit a common border are aggregated.

\begin{tabular}{|c|c|c|c|c|}
\hline & & & Left & Right \\
\hline & Region name & NVA (\%) & $x, y, z(m m)$ & $x, y, z(m m)$ \\
\hline Left $>$ Right & G_Frontal_Sup-1 & 139 & $-16,65,13$ & $13,68,11$ \\
\hline & S_Postcentral-3 & 118 & $-43,-33,44$ & $48,-26,43$ \\
\hline & S_Superior_Temporal-1 & 117 & $-50,14,-22$ & $52,13,-26$ \\
\hline & G_Temporal_Pole_Mid-1 & 70 & $-45,7,-34$ & $48,8,-33$ \\
\hline & G_SupraMarginal-7 & $\mathbf{8 3}$ & $-55,-52,26$ & $55,-46,33$ \\
\hline & S_Superior_Frontal-4 & 79 & $-23,29,47$ & $20,36,48$ \\
\hline & S_Inferior_Frontal-1 & 70 & $-44,38,12$ & $46,40,10$ \\
\hline & G_Supp_Motor_Area-3 & 67 & $-7,8,64$ & $6,10,65$ \\
\hline Right $>$ Left & G_Temporal_Pole_Mid-2 & -124 & $-35,9,-33$ & $35,12,-34$ \\
\hline & Caudate-7 & -91 & $-18,-12,25$ & $17,-8,24$ \\
\hline & S_Superior_Frontal-1 & -79 & $-22,61,-8$ & $20,63,-6$ \\
\hline & G_Frontal_Mid-5 & -73 & $-43,20,37$ & $42,17,41$ \\
\hline & G_Frontal_Mid-3 & -71 & $-39,31,35$ & $37,33,35$ \\
\hline & G_Frontal_Mid-1 & -61 & $-40,41,20$ & $41,44,13$ \\
\hline & G_Parietal_Inf-1 & -72 & $-45,-53,49$ & $43,-53,48$ \\
\hline & G_Cingulum_Post-2 & -69 & $-4,-39,27$ & $8,-43,31$ \\
\hline & G_Parietal_Sup-1 & -62 & $-24,-47,59$ & $24,-47,62$ \\
\hline & S_Superior_Temporal-2 & -62 & $-55,-7,-13$ & $54,-2,-15$ \\
\hline
\end{tabular}

In the frontal lobe, the middle frontal regions were rightward asymmetrical, whereas the superior frontal sulcus, superior frontal gyrus and inferior frontal sulcus regions exhibited leftward asymmetry. In the medial wall, the largest subpart of SMA (G_Supp_Motor_Area-3) was leftward asymmetrical.

The G_Temporal_Pole_Mid-1 was larger in the left compared with right hemisphere, whereas the reverse was observed for the neighboring G_Temporal_Pole_Mid-2 and S_Superior_Temporal-2 regions.

In the parietal cortex, the S Postcentral-3 was larger on the left, whereas the neighboring G_Parietal_Inf-1 exhibited the reverse asymmetry. Note that the G_SupraMarginal-7 was larger on the left without any neighboring ROI exhibiting the reverse asymmetry.

\subsubsection{Regional functional homotopic correlation}


A 3-D rendering of regional average homotopic correlation (AHC) values is presented on Fig. 4A. The regional AHC values range from 0.26 to 0.84 . The highest $\mathrm{AHC}$ values were observed on the medial face in regions surrounding the corpus callosum. The highest correlations on the cortical convexity were observed in the cortex surrounding the Rolando sulcus, insula, temporal superior gyrus, supramarginal gyrus and occipital cortices. Most of the lateral frontal and temporal cortices (except for the superior temporal cortex) showed the weakest correlations. Additional intermediate correlations values were found in the inferior frontal and parietal regions.

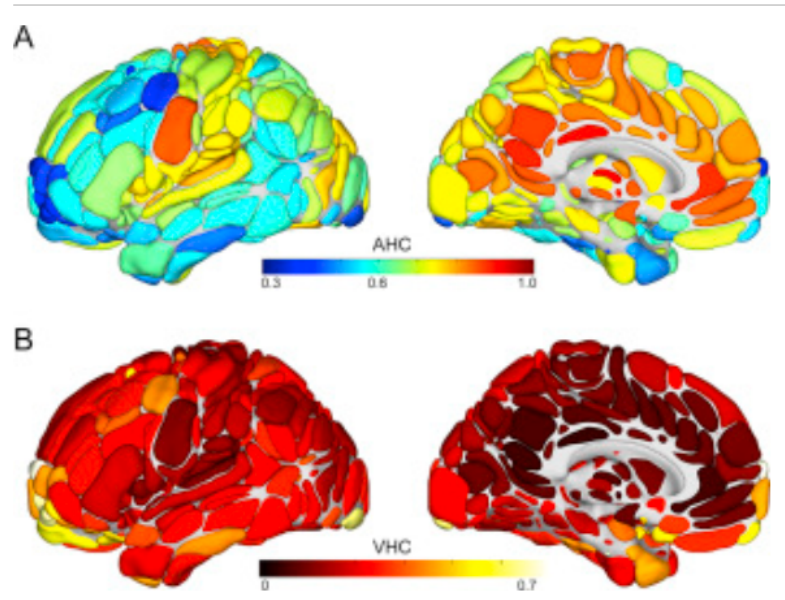

Download : Download full-size image

Fig. 4. Analysis of the AICHA homotopic correlation. (A) Regional average homotopic correlation (AHC) 3-Dimensional rendering map. (B) Regional variation coefficient homotopic correlation (VHC) 3-Dimensional rendering map.

The coefficient of variation of the homotopic correlation strength (VHC) is shown in Fig. 4B. Regional values look homogeneous across the brain with some high outlier values located mostly in the lower parts of the brain. BOLD signal susceptibility artifacts that are important when acquiring the whole brain may explain the highest variability in those regions. Indeed, the frontal lobe included most of those outliers: G_Frontal_Sup-1-L, S_Sup_Frontal-1, G_Frontal_Sup_Orb-1-L, G_Frontal_Inf_Orb-2, G_Frontal_Mid_Orb-1, S_Orbital-1, G_Frontal_Med_Orb-1 and $\bar{S}$ _Sup_Frontal-2. Other areas were located in (from the front to the back of the brain): G_Insula-anterior-1, G_ParaHippocampal-3, G_Temporal_Pole_Mid2, G_Temporal_Pole_Mid-3, G_Fusiform-2 and G_Occipital_Pole-1. $\overline{\text { In }}$ the upper parts of the brain only the G_Frontal_Sup-3 showed similar high value.

\subsection{Comparison of AICHA ROI homogeneity with other atlases}

For each atlas, the regional functional homogeneity was averaged across the subjects and presented as a boxplot in Fig. 5. 


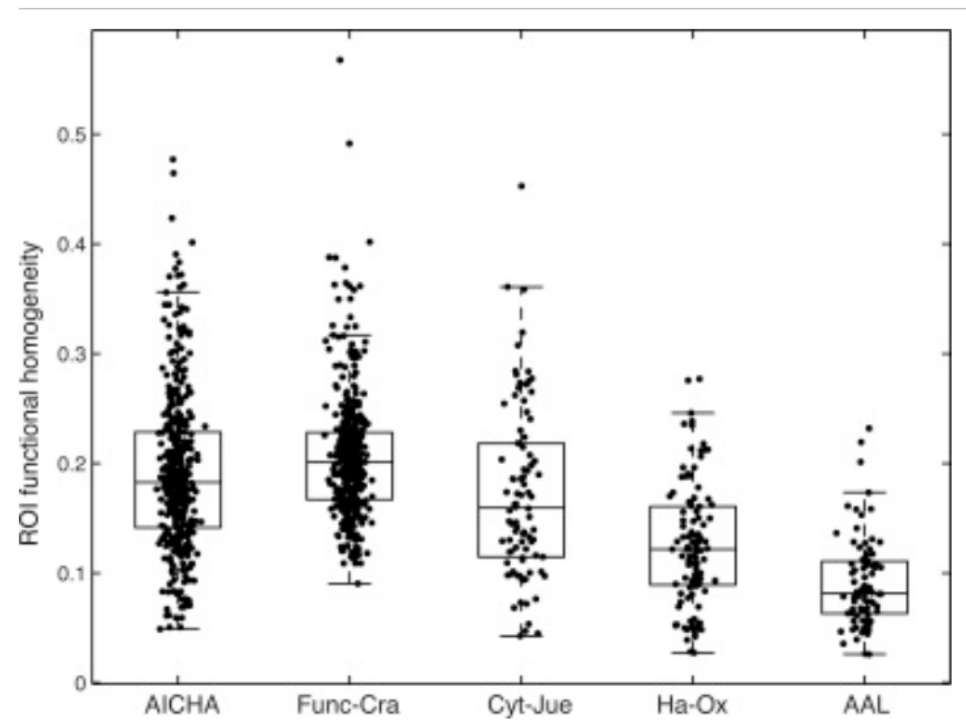

Download : Download full-size image

Fig. 5. Regional functional homogeneity of 5 atlases. AICHA and Craddock parcellation are based on functional landmarks, Juelich parcellation is based on the cytoarchitectonic borders, and Harvard-Oxford and AAL parcellations are shaped by macroscopic anatomical landmarks. Homogeneity is computed using database 2 (average of 88 subjects). Each boxplot shows individual regional values, the median and both the $25^{\text {th }}$ and $75^{\text {th }}$ percentiles together with the outlier limits that approximately correspond to \pm 2.7 standard deviations of the median value.

The median regional homogeneity was higher for the Func-Cra atlas $(0.20)$ followed sequentially by AICHA (0.18), Cyt-Jue (0.16), Ha-Ox (0.12) and AAL (0.08).

The regional homogeneity was significantly higher in Func-Cra compared with AICHA (student t-test $\mathrm{t}(778)=2.96, \mathrm{p}=0.003$ ), and these two atlases exhibited a larger regional functional homogeneity compared with the 2 anatomical atlases $(t>8.86, p<10-16)$.

The cytoarchitectonic atlas (Cyt-Jue) homogeneity was below the AICHA functional atlas homogeneity $(\mathrm{t}(472)=2.16, \mathrm{p}=0.03)$; however, this finding must be confirmed because the former atlas provides only a partial coverage of the gray matter.

\subsection{Descriptive pattern of the regional functional correlation}

The categorical distribution of functional correlation connections at increasing sparsity thresholds is presented in Fig. 6A. We first observe a preponderance of the homotopic categories. With a sparsity threshold of $2 \%$ (noted St 2 ), $62 \%$ of the connections were homotopic. This proportion of homotopic connections remained preponderant up to a threshold at St12, where a balanced proportion of homotopic (27.6\%), left intra-hemispheric (27.4\%) and right hemispheric connections (27.9\%) was observed (Fig. 6A, left panel). Note that at this threshold, 108 of the 192 potential homotopic connections (Fig. 6A right panel) were above the sparsity threshold in most subjects. 

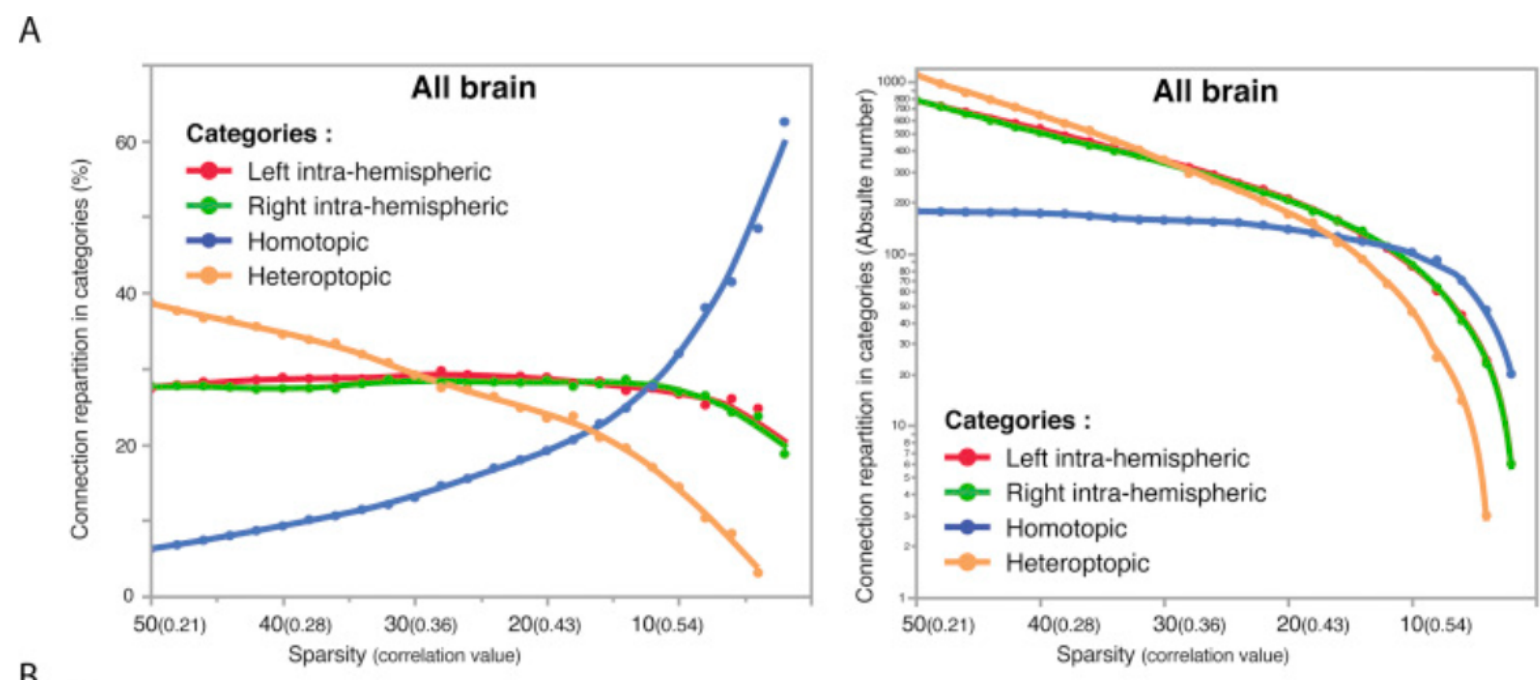

B
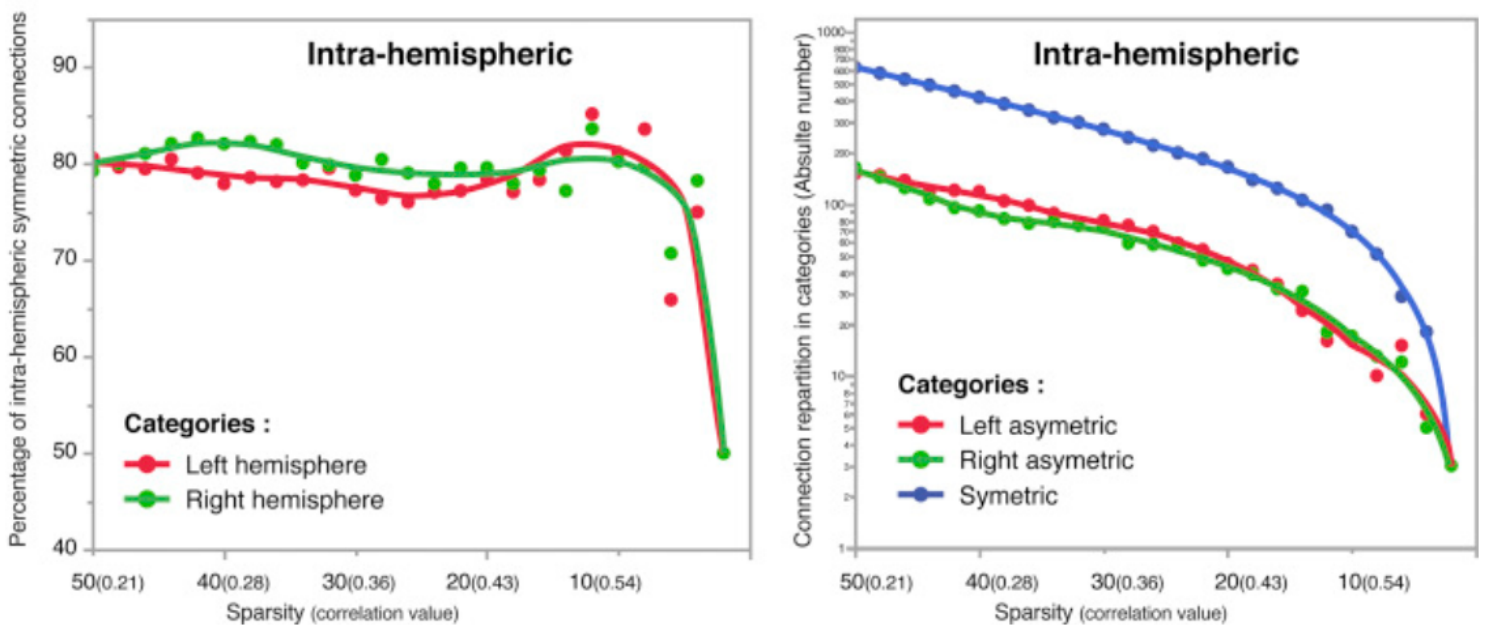

Download : Download full-size image

Fig. 6. Functional connection categorical sparsity level analysis. (A) Left side: Repartition of the connections in each category in the percentage of the total number of connections identified at various sparsity thresholds. The 4 categories include: the intra-hemispheric left/right connections and the inter-hemispheric homotopic/heterotopic connections. Right side: idem than left side but shows the absolute number of connections. (B) Left side: Number of intra-hemispheric symmetric connections (i.e., identified between the same 2 regions of the left and right hemispheres accordingly to the homotopic description) in the percentage of the number of intra-hemispheric connections in each hemisphere. Right side: Absolute number of connections among the symmetric and asymmetric (identified in only one hemisphere) intra-hemispheric categories.

The intra-hemispheric right and left proportions were comparable across all sparsity thresholds (Fig. 6A). 
More than $50 \%$ of the intra-hemispheric connections were bilateral, and the proportion was stable at $80 \%$ below St10 (Fig. 6B).

An example of the pattern of regional connections at threshold St30 for inter-hemispheric and intra-hemispheric categories is provided in Fig. 7, Fig. 8, respectively.

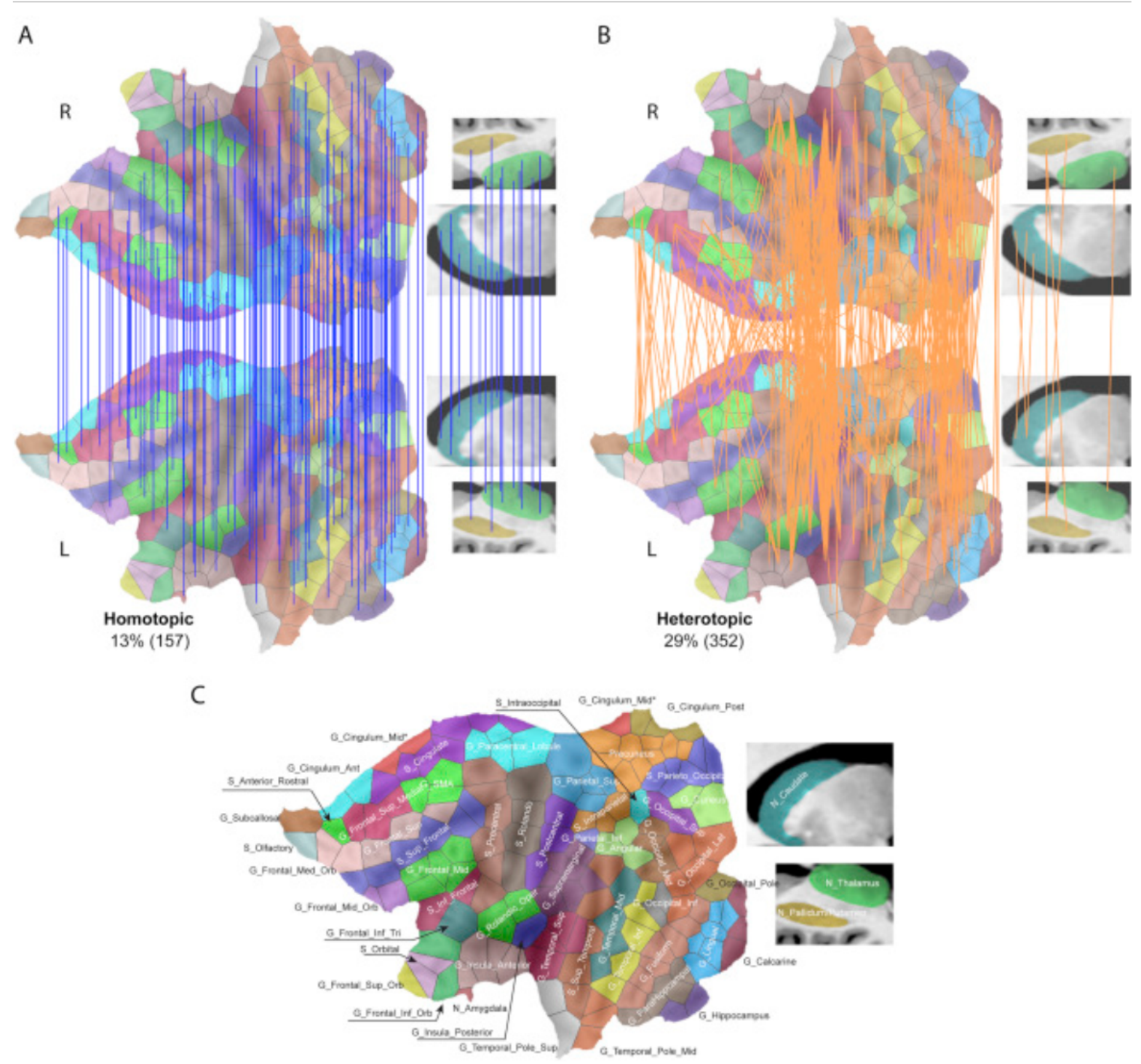

Download : Download full-size image

Fig. 7. Inter-hemispheric connections. Homotopic (A) and heterotopic (B) connections identified at a sparsity threshold of $30 \%$. The category percentage and absolute number of connections are listed. Data are presented on the flat map of the AICHA atlas (C). 

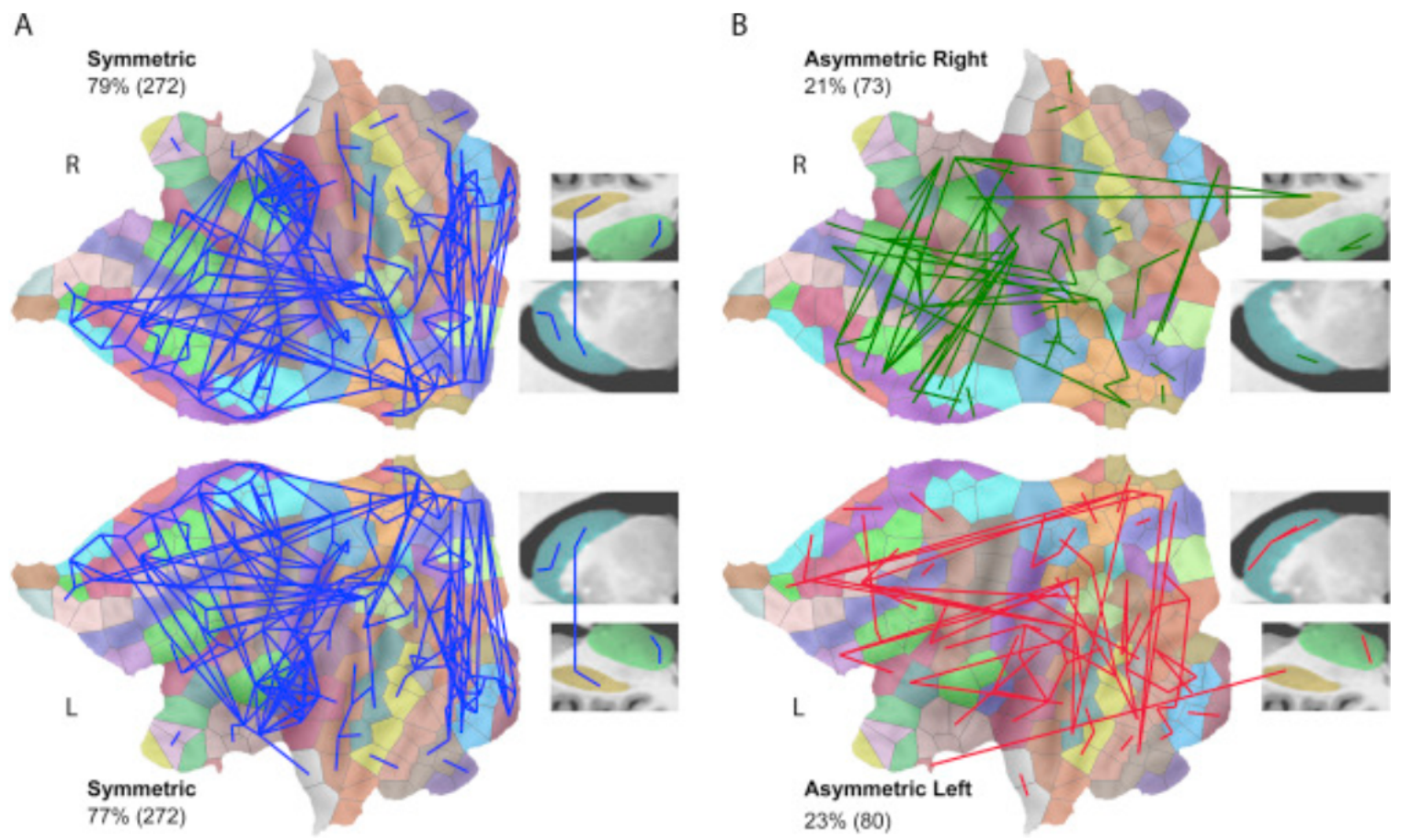

Download : Download full-size image

Fig. 8. Intra-hemispheric connections. Symmetric (A) and asymmetric (B) connections identified at a sparsity threshold of $30 \%$. The category percentage and absolute number of connections are listed. Data are presented on the flat map of the AICHA atlas.

\section{Discussion}

We will discuss the choices we made in the construction of AICHA, including the advantages and potential limitations of these choices.

\subsection{Large population dataset balanced for handedness}

The issue of the dataset used to define an atlas is often overlooked. Very popular atlases, such as the Talairach atlas (Talairach and Tournoux, 1988), the Brodmann atlas (Brodmann and Garey, 1994) and AAL (Tzourio-Mazoyer et al., 2002), are based on a single individual and/or a single hemisphere. The increasing ability to obtain access to in-vivo individual data makes it possible to consider brain variability in atlas construction by including several brains, which raises questions regarding the selection of the sample participants. The question then arises of the sample size and whether the sample should include participants with specific demographic/phenotypic characteristics, if it should be balanced for some of these characteristics, or if it should be randomly constituted from the population. There is no consensus in the literature; the sample size ranges from one (see previous discussion) to tens of individuals (37-79, Desikan et al., 2006, Craddock et al., 2012, Shen et al., 2013), including one atlas built from 1000 subjects (Yeo et al., 2011). Most atlases are matched for gender, whereas other phenotypes are uncontrolled. For example, of the 4 atlases previously 
discussed, only Shen et al. (2013) reported a handedness phenotype (only right handed subjects were selected). Additionally, the age range of the participants is highly variable from 18 years for the lower bound (in general, based on the legal majority) to approximately 50/55 years for the upper bound; however, the age distribution is typically not uniform because younger subject recruitment is typically more common.

We chose to build AICHA from a large set of 281 healthy volunteers that covered a consistent range of variability in age and cultural levels. We balanced our sample for gender and handedness because these two factors are known to have strong impacts on anatomical and functional brain organization and asymmetries. Sex impacts brain anatomy because of the differences in brain volume (Leonard et al., 2008, Luders et al., 2009, Inano et al., 2013), as well as brain white matter connectivity (Ingalhalikar et al., 2014) and function (for a review, see Sacher et al., 2013). Handedness is also known to modify the anatomo-functional brain support of motor functions (Amunts et al., 2000, Amunts et al., 1996, Sun et al., 2012, Herve et al., 2006), as well as other lateralized cognitive functions, such as language (review in Hervé et al., 2013). We believe that it was important to avoid the bias induced by the inclusion of only $10 \%$ of left-handers (prevalence of left handers in the general population) to avoid the drawback of the under representation of rare brain organizations, such as reversed language lateralization (e.g., Mazoyer et al., 2014).

We could have utilized random sampling from the general population, which is the theoretical best solution, but it is hard to accomplish this approach in practice because this sampling would lead to the inclusion of subjects with neurological disorders or brain lesions.

Furthermore, restriction of the sampled population to "normal" subjects would raise the unresolvable issue of defining normality.

The option that consists of ad hoc sampling, which is based on specific criteria, for example, only right-handed men, would lead to the creation of one atlas for each different combination of characteristics; this procedure is combinatory and can rapidly increase the number of atlases. Furthermore, it imposes the classification of participants according to the set of chosen properties; thus, this may result in a difficult choice regarding which atlas to use (for example, with ambilateral subjects when 2 atlases are created for right and left handers, respectively). Finally, it may be difficult to compare results obtained in groups analyzed with different atlases because their regional homology will not be known.

\subsection{Volumetric normalization space}

We choose to develop AICHA in the volumetric space to benefit from the substantial amount of structured information regarding the functional anatomy of cognitive functions that is available in this framework. Spatial normalization of individual data in a common space has initially been developed using a volumetric approach (Ashburner and Friston, 1999; see also review Evans et al., 2012), and this normalization has proven to be a successful means for comparing different studies in a coordinate reference system, such as the Talairach or Montreal Neurological Institute (MNI) spaces. The development of this procedure has enabled the design of large databases (Brainmap, Fox et al., 2005b) that compile extensive functional localizations of cognitive functions (currently 2466 papers) and make it available to the community (e.g., Toro et al., 2008). The methodological evolution based on gray matter surface normalization (Dale et al., 1999) provides more sensitive approaches to process data (Jo et al., 2007) and offers a better handling of the partial volume effect. Despite a more limited corpus of research published compared with volumetric normalization, surface normalization has been adopted by some leading projects (e.g., the Human connectome 
project, Glasser et al., 2013). In the near future, we will make a surface-based version of AICHA available.

\subsection{Atlas landmarks}

AICHA construction was based on resting state fMRI data homogeneity, which enabled us to set the number of regions at a desired value. The limited number of sulci consistent across individuals restricts the anatomical parcellation to a limited ROI number, such as 45 ROIs per hemisphere for AAL (cerebrum region parcellation in gyri/nuclei, Tzourio-Mazoyer et al., 2002) or 74 ROIs per hemisphere in the Destrieux atlas (cerebrum region parcellation in gyri/sulci/nuclei, Destrieux et al., 2010). In addition, anatomical atlases exhibit important differences in ROI size, including very large ROIs, such as the middle temporal in AAL, because of the absence of a reproducible anatomical marker to enable a finer grain parcellation. Arbitrary geometric criteria are then applied, which vary across atlases, such as the cingulum in AAL (three parts) or the frontal pole in the Ha-Ox atlas (Desikan et al., 2006).

AICHA parcellation is based on the time correlation structure of the resting state functional signal, which is a low frequency BOLD pseudo-oscillatory activity that subtends intrinsic brain function (Doucet et al., 2011, Fox et al., 2005a, Golland et al., 2008). It has been demonstrated that the regional assemblies evidenced by intrinsic connectivity analyses are comparable to the networks that subtend extrinsic or goal directed tasks (Laird et al., 2011, Smith et al., 2009). More recent work demonstrates that the brain's functional network architecture during task performance is primarily shaped by the resting state network architecture while showing also some small differences (Cole et al., 2014) also reported by Mennes et al. (2013) in some areas. Thus, an atlas parcellation based on intrinsic connectivity of regional assemblies is meaningful not only for the study of intrinsic connectivity networks but also possibly for the investigations of the neural substrates of cognitive functions.

\subsection{Atlas building}

The algorithmic building of AICHA is based on a 4 stage procedure, including ICA based group analysis, k-means clustering, homotopy driven parcellation refinement and labeling.

The first stage of the analysis is rooted in the observation that the correlation strength of the anatomically defined homotopic connections largely predominates over the heterotopic connections (Stark et al., 2008). Using an ICA based analysis, this organization led to a partition in resting state networks that exhibited the gross symmetric organization characteristic of homotopy for a majority of these networks (Beckmann et al., 2005).

ICA applied to a group of subjects (Beckmann et al., 2005, Calhoun et al., 2001) has been shown to generate a brain parcellation in spatially resting state networks that are highly reproducible across many laboratories and datasets (e.g., Chen et al., 2008, Damoiseaux et al., 2006, Kiviniemi et al., 2009, Varoquaux et al., 2010). For this first stage, we choose the implementation of the group ICA (MICCA, Naveau et al., 2012) based on a classification of individual ICAs because of the following advantages: it could handle the massive multisubject data involved in AICHA definition, and it has been demonstrated to lead to reproducible whole-brain parcellation. Independent processing of the two halves of the dataset demonstrated that the extracted RSNs encompass $98 \%$ of the cerebrum gray matter (Naveau et al., 2012). The MICCA algorithm also has the advantage of filtering out the non-spatially reproducible artifactual components that are commonly observed in the individual ICA analysis. 
The second stage, i.e., the k-means, was imposed by the outcome of the group ICA analysis, which does not preclude a voxel to belong to two or more RSNs. The detection of a voxel that belongs to 2 or more regions is a meaningful observation for a neuroscientist, which corresponds to the concept of the "hub" regions (Achard et al., 2006) that are believed to be essential for the flow of information between the networks (Bullmore and Bassett, 2011). However, a voxel that belongs to 2 or more regions is an undesirable feature in an atlas. For example, in the case of functional connectivity analysis, it will create a built-in link between the regions independent of the measured signal. To avoid this feature, we entered the RSN functional connectivity matrices into the classification algorithm.

We selected k-means because it does not impose geometrical constraints on the ROIs as opposed to other algorithms, such as the Normalized-Cut, which constrains parcels to have more or less equivalent sizes and shapes (Craddock et al., 2012, Shen et al., 2013). This is a crucial point because as a result of the extremely convoluted shape of the brain, there is no reason to assume that macroscopic functional units have geometrically equivalent shapes.

As previously discussed, in anatomical atlases, the number of population reproducible sulci determines the number of parcels; however, in functional atlases, the number of parcels is a user choice. Other authors have published atlases at different resolutions: 7 and 17 networks per hemisphere for Yeo et al. (2011), 50/100/150 regions per hemisphere for Shen et al. (2013) and 50 to 1000 regions (in increments of 50) for Craddock et al. (2012). For AICHA, while a large range of k-level values was present, we used a heuristic criterion based on the robustness of the parcellation to set the optimal levels of partition. The most robust candidates were obtained with a brain parcellation between $\mathrm{k}-\mathrm{level}=100$ and $\mathrm{k}$-level $=175$ parcels. $\mathrm{We}$ choose the level with the highest number of parcels to stay in the optimal range while using an aggregative (i.e., decreasing the number of parcels) procedure to uncover the homotopic organization.

The third stage comprised the homotopy driven parcellation refinement. Note that prior to refinement, there was an important amount of homotopic regions $(60 \%)$, i.e., regions that fulfill the maximal correlation criteria. For the $40 \%$ remaining regions, regarding the arbitrariness of the number of clusters, it appeared reasonable to modify the number of regions by separation or aggregation until we reached the desired features. We chose to implement an aggregation procedure because it was less arbitrary than a random based regional separation procedure. This procedure was successful as we attained $93 \%$ regional homotopy and $99 \%$ after segmentation artifact corrections. Such a high level was expected because we based our atlas construction on the existence of a predominant functional homotopic correlation between the two hemispheres (Stark et al., 2008). Nevertheless, it does not imply that the two hemispheres are identical because we observed regional size differences between ROIs of a homotopic pair that increased to $140 \%$. Interestingly, the frontal lobe comprises the majority of the highest volumetric asymmetries. This finding could be further studied in the framework of the hemispheric specialization (Hervé et al., 2013). For example, the larger frontal regions in the left hemisphere could be related to the leftward asymmetry observed during language processing (Vigneau et al., 2006, Vigneau et al., 2010). Finally, note that the homotopic stage is independent of the two first stages and thus could be applied to any existing atlas.

As previously shown by Stark et al. (2008), we observed that the primary cortices showed the highest homotopic correlation strength and the heteromodal cortices the lowest. According to 
Mesulam's model, these areas cover the two extremes of the proposed hierarchical classification that are the primary areas and the heteromodal association areas, respectively (Mesulam, 2000).

\subsection{Comparison of regional homogeneity on different atlases}

As expected, the ROI functional homogeneity analysis (which quantifies the similarity of the resting state signals within each region) demonstrates a clear cut-off between the 2 functional based atlases and the two anatomical based atlases, which favors the former atlases. This was expected because both ICA-k means (AICHA) and Normalized-CUT (Func-Cra) methodologies were chosen to maximize this feature. Between these 2 atlases, Func-Cra exhibits a higher homogeneity. As previously discussed, this finding was expected as the spatial constraint of the N-Cut parcellation scheme favors more compact patches.

Note that we have restricted our analysis to the intra-regional homogeneity and not to a criterion taking into account both intra- and the inter-regional connectivity. Indeed, the known mixing of high and low regional functional connectivity strength in the brain (see for example, Stark et al., 2008) will make the inter-regional connectivity complex to interpret in this framework.

\subsection{AICHA as a tool to investigate brain functional asymmetries}

Recent renewed interest in the hemispheric specialization (Herve et al., 2013) has emphasized the need to categorize functional connections as inter-hemispheric, either homotopic (Stark et al., 2008, Zuo et al., 2010) or heterotopic (Gee et al., 2011, Liu et al., 2009), or intrahemispheric (Iturria-Medina et al., 2011). AICHA provides a categorization at a regional level of sampling that is more precise than the anatomical atlas level; however, it is less precise than the voxel level. However, at the voxel level, the dimensionality of potential connections is difficult to handle in connectivity and graph approaches.

The sparsity analysis completed with AICHA highlighted the major symmetric organization of the brain. This symmetrical organization was present at the inter-hemispheric level between homotopic regions (as shown by others, Stark et al., 2008); this result was expected because of the principles that underlie AICHA elaboration. Interestingly, however, the symmetry was also evidenced by a similar proportion of right and left intra-hemispheric connections. We also described a heterotopic connection that had, per construction, lower correlation values and a distribution that favored the homoareal connections defined as callosal connections between non-homotopic contralateral sites close to each homologue (Kaiser, 2005). An exploration of different levels of sparsity indicated that heterotopic connections were preponderant within the insula and the lower part of the occipital lobe. Whether such distribution is due to instrumental factors, such as partial volume effect, or regional differences in organization will be the object of further investigations.

Beyond the matching level of right and left intra-hemispheric connections, we observed that the level of symmetrical connections reached a plateau at $80 \%$, which was below an $8 \%$ sparsity threshold. This observation reinforces the similarity feature of the two hemispheres. Interestingly, it appears that higher correlation values of long range connections are observed in two approximately orthogonal directions: the antero-posterior dimension between the frontal and parietal lobes and the right-left dimension between the medial frontal lobe toward the insula and lateral frontal inferior lobes. These high correlations in selective orientations fit with Zilles proposal of "unifying principles behind the structural complexity of the cerebral cortex" according to the gradation hypothesis, genetic topography and cytoarchitecture (Zilles 
and Amunts, 2012). The $20 \%$ of asymmetric connections are interesting connections to study in the framework of hemispheric specialization.

\subsection{How to obtain and use AICHA}

AICHA is provided in a "nifti" image format with additional files for use with the AAL toolbox (Tzourio-Mazoyer et al., 2002), mricron

(http://www.mccauslandcenter.sc.edu/mricro/mricron) software and FSLView atlas tools (http://fsl.fmrib.ox.ac.uk/fsl/fslview/).

For region interest analysis, we also provide an anatomical labeling of AICHA. This labeling is based on AAL and was adapted to consider the specificity of AICHA. We created a sulcal regional label (labeled S-) that groups regions that encompass one or two banks of the cortex buried in a specific sulcus. Regions that encompass both gyral and sulcal matter were labeled, as in the original AAL definition, as the gyral region. Note also that only a small part of the existing sulci are identified in the AAL partition. This factor explains that a majority of voxels belongs to gyral matter. Similar to AAL, the labeling provides a coarse localization to facilitate data interpretation, but it cannot be considered as a precise measure of gyral versus sulcal gray matter; this distinction is unreachable given the resolution of functional atlases, such as AICHA.

It is important to underline that over $80 \%$ of the regions corresponded to a single anatomical AAL label, either a gyrus (labeled G-) or a nucleus (labeled N-), although no anatomical landmarks were used in the atlas construction. This finding may represent a heuristical demonstration that the combination of the low resolution BOLD EPI acquisition with the partial volume effects that occur between the two banks of a sulcus does not lead to an artefactual mixing of functional information. One may thus consider that AICHA provides a functionally driven sub-partition of anatomical ROIs, which is thus a tool for finer grain analyses of numerous previous results obtained using AAL and graph analysis of resting state data. In this framework, using AICHA, we demonstrated that language lateralization was associated with differences in inter-hemispheric connectivity during resting state by measuring their regional homotopic inter-hemispheric intrinsic connectivity coefficient in 36 ROIs implicated in language processing and known to be connected via the corpus callosum (Tzourio-Mazoyer et al., 2015).

\section{Conclusion}

We have developed an atlas of ROIs that covers the entire cerebral gray matter using a parcellation scheme based on the intrinsic connectivity of homotopic areas. Considering that AICHA was developed from resting state low frequency BOLD pseudo-oscillatory activity, which is known to subtend intrinsic brain function and likely task performance, we believe that this atlas will be useful for connectivity and graph analysis of both resting-state and taskrelated functional imaging data. In addition, the fact that the hemispheric parcellation of regions is based on the strength of homotopic intrinsic correlations indicates it is a new and powerful tool for the investigation of regional asymmetries and thus brain hemispheric lateralization. The AICHA atlas is available on a mail request to the corresponding author. 


\section{Acknowledgments}

We thank Guy Perchey for his essential help with data acquisition.

\section{Appendix A. Supplementary data}

\section{References}

S. Achard, R. Salvador, B. Whitcher, J. Suckling, E. Bullmore A resilient, low-frequency, small-world human brain functional network with highly connected association cortical hubs. J Neurosci, 26 (2006), pp. 63-72

K. Amunts, L. Jancke, H. Mohlberg, H. Steinmetz, K. Zilles Interhemispheric asymmetry of the human motor cortex related to handedness and gender. Neuropsychologia, 38 (2000), pp. 304-312

K. Amunts, G. Schlaug, A. Schleicher, H. Steinmetz, A. Dabringhaus, P.E. Roland, et al. Asymmetry in the human motor cortex and handedness. NeuroImage, 4 (1996), pp. 216-222

J. Ashburner, K.J. Friston Nonlinear spatial normalization using basis functions. J Acoust Soc Am, 106 (1999), pp. 449-457

J. Ashburner, K.J. Friston Unified segmentation; NeuroImage, 26 (2005), pp. 839-851

C.F. Beckmann, M. DeLuca, J.T. Devlin, S.M. Smith Investigations into resting-state connectivity using independent component analysis. Philos Trans R Soc Lond, B: Biol Sci, 360 (2005), pp. 1001-1013

B. Biswal, F.Z. Yetkin, V.M. Haughton, J.S. Hyde Functional connectivity in the motor cortex of resting human brain using echo-planar MRI. Magn Reson Med, 34 (1995), pp. 537541

K. Brodmann, L.J. Garey Brodmann's 'localisation in the cerebral cortex'. Smith-Gordon, London (1994)

E.T. Bullmore, D.S. Bassett Brain graphs: graphical models of the human brain connectome. Annu Rev Clin Psychol, 7 (2011), pp. 113-140

V.D. Calhoun, T. Adali, G.D. Pearlson, J.J. Pekar A method for making group inferences from functional MRI data using independent component analysis. Hum Brain Mapp, 14 (2001), pp. 140-151

S. Caspers, S. Geyer, A. Schleicher, H. Mohlberg, K. Amunts, K. Zilles The human inferior parietal cortex: cytoarchitectonic parcellation and interindividual variability. NeuroImage, 33 (2006), pp. 430-448 
S. Chen, T.J. Ross, W. Zhan, C.S. Myers, K.S. Chuang, S.J. Heishman, et al. Group independent component analysis reveals consistent resting-state networks across multiple sessions. Brain Res, 1239 (2008), pp. 141-151

M.W. Cole, D.S. Bassett, J.D. Power, T.S. Braver, S.E. Petersen Intrinsic and task-evoked network architectures of the human brain. Neuron, 83 (2014), pp. 238-251

R.C. Craddock, G.A. James, P.E. Holtzheimer 3rd, X.P. Hu, H.S. Mayberg A whole brain fMRI atlas generated via spatially constrained spectral clustering. Hum Brain Mapp, 33 (2012), pp. 1914-1928

A.M. Dale, B. Fischl, M.I. Sereno Cortical surface-based analysis. I. Segmentation and surface reconstruction. NeuroImage, 9 (1999), pp. 179-194

J.S. Damoiseaux, S.A. Rombouts, F. Barkhof, P. Scheltens, C.J. Stam, S.M. Smith, et al. Consistent resting-state networks across healthy subjects. Proc Natl Acad Sci USA, 103 (2006), pp. 13848-13853

R.S. Desikan, F. Segonne, B. Fischl, B.T. Quinn, B.C. Dickerson, D. Blacker, et al. An automated labeling system for subdividing the human cerebral cortex on MRI scans into gyral based regions of interest. NeuroImage, 31 (2006), pp. 968-980

C. Destrieux, B. Fischl, A. Dale, E. Halgren Automatic parcellation of human cortical gyri and sulci using standard anatomical nomenclature. NeuroImage, 53 (2010), pp. 1-15

G. Doucet, M. Naveau, L. Petit, N. Delcroix, L. Zago, F. Crivello, et al. Brain activity at rest: a multiscale hierarchical functional organization. J Neurophysiol, 105 (2011), pp. 2753-2763

A.C. Evans, A.L. Janke, D.L. Collins, S. Baillet Brain templates and atlases. NeuroImage, 62 (2012), pp. 911-922

M.D. Fox, A.Z. Snyder, J.L. Vincent, M. Corbetta, D.C. Van Essen, M.E. Raichle The human brain is intrinsically organized into dynamic, anticorrelated functional networks. Proc Natl Acad Sci USA, 102 (2005), pp. 9673-9678

P.T. Fox, A.R. Laird, S.P. Fox, P.M. Fox, A.M. Uecker, M. Crank, et al. BrainMap taxonomy of experimental design: description and evaluation. Hum Brain Mapp, 25 (2005), pp. 185-198

J.K. Fuster Linkage at the top. Neuron, 21 (1998), pp. 1223-1229

D.G. Gee, B.B. Biswal, C. Kelly, D.E. Stark, D.S. Margulies, Z. Shehzad, et al. Low frequency fluctuations reveal integrated and segregated processing among the cerebral hemispheres. NeuroImage, 54 (2011), pp. 517-527

M.F. Glasser, S.N. Sotiropoulos, J.A. Wilson, T.S. Coalson, B. Fischl, J.L. Andersson, et al. The minimal preprocessing pipelines for the Human Connectome Project. NeuroImage, 80 (2013), pp. 105-124 
Y. Golland, P. Golland, S. Bentin, R. Malach Data-driven clustering reveals a fundamental subdivision of the human cortex into two global systems. Neuropsychologia, 46 (2008), pp. $540-553$

P.Y. Herve, F. Crivello, G. Perchey, B. Mazoyer, N. Tzourio-Mazoyer Handedness and cerebral anatomical asymmetries in young adult males NeuroImage, 29 (2006), pp. 1066-1079

P.Y. Herve, L. Zago, L. Petit, B. Mazoyer, N. Tzourio-Mazoyer Revisiting human hemispheric specialization with neuroimaging. Trends Cogn Sci, 17 (2013), pp. 69-80

J. Hill, D. Dierker, J. Neil, T. Inder, A. Knutsen, J. Harwell, et al. A surface-based analysis of hemispheric asymmetries and folding of cerebral cortex in term-born human infants. $\mathrm{J}$

Neurosci, 30 (2010), pp. 2268-2276

J. Himberg, A. Hyvarinen, F. Esposito Validating the independent components of neuroimaging time series via clustering and visualization. NeuroImage, 22 (2004), pp. 12141222

L. Hubert, P. Arabie Comparing partitions. J Classif, 2 (1985), pp. 193-218

S. Inano, H. Takao, N. Hayashi, N. Yoshioka, H. Mori, A. Kunimatsu, et al. Effects of age and gender on neuroanatomical volumes. J Magn Reson Imaging, 37 (2013), pp. 1072-1076

M. Ingalhalikar, A. Smith, D. Parker, T.D. Satterthwaite, M.A. Elliott, K. Ruparel, et al. Sex differences in the structural connectome of the human brain. Proc Natl Acad Sci USA, 111 (2014), pp. 823-828

Y. Iturria-Medina, A. Perez Fernandez, D.M. Morris, E.J. Canales-Rodriguez, H.A. Haroon, L. Garcia Penton, et al. Brain hemispheric structural efficiency and interconnectivity rightward asymmetry in human and nonhuman primates. Cereb Cortex, 21 (2011), pp. 56-67

H.J. Jo, J.M. Lee, J.H. Kim, Y.W. Shin, I.Y. Kim, J.S. Kwon, et al. Spatial accuracy of fMRI activation influenced by volume- and surface-based spatial smoothing techniques.

NeuroImage, 34 (2007), pp. 550-564

H.J. Jo, Z.S. Saad, S.J. Gotts, A. Martin, R.W. Cox Quantifying agreement between anatomical and functional interhemispheric correspondences in the resting brain. PLoS ONE, 7 (2012), p. e48847

M. Joliot, B.M. Mazoyer IEEE (Ed.), 3-D segmentation, interpolation and delineation of cortical gyri on MRI data, IEEE, Santa Fe, NM (1991), pp. 2118-2123

D.A. Kaiser Synchrony measures and non-homotopic: do synchrony measures between nonhomotopic areas make sense? J Neurother., 9 (2005), pp. 97-108

D.N. Kennedy, N. Lange, N. Makris, J. Bates, J. Meyer, V.S. Caviness Jr. Gyri of the human neocortex: an MRI-based analysis of volume and variance. Cereb Cortex, 8 (1998), pp. 372384 
V. Kiviniemi, T. Starck, J. Remes, X. Long, J. Nikkinen, M. Haapea, et al. Functional segmentation of the brain cortex using high model order group PICA. Hum Brain Mapp, 30 (2009), pp. 3865-3886

A.R. Laird, P.M. Fox, S.B. Eickhoff, J.A. Turner, K.L. Ray, D.R. McKay, et al. Behavioral interpretations of intrinsic connectivity networks. J Cogn Neurosci, 23 (2011), pp. 4022-4037

C.M. Leonard, S. Towler, S. Welcome, L.K. Halderman, R. Otto, M.A. Eckert, et al. Size matters: cerebral volume influences sex differences in neuroanatomy. Cereb Cortex, 18 (2008), pp. 2920-2931

H. Liu, S.M. Stufflebeam, J. Sepulcre, T. Hedden, R.L. Buckner Evidence from intrinsic activity that asymmetry of the human brain is controlled by multiple factors. Proc Natl Acad Sci USA, 106 (2009), pp. 20499-20503

E. Luders, C. Gaser, K.L. Narr, A.W. Toga Why sex matters: brain size independent differences in gray matter distributions between men and women. J Neurosci, 29 (2009), pp. $14265-14270$

O.C. Lyttelton, S. Karama, Y. Ad-Dab'bagh, R.J. Zatorre, F. Carbonell, K. Worsley, et al. Positional and surface area asymmetry of the human cerebral cortex. NeuroImage, 46 (2009), pp. $895-903$

B. Mazoyer, L. Zago, G. Jobard, F. Crivello, M. Joliot, G. Perchey, et al. Gaussian mixture modeling of hemispheric lateralization for language in a large sample of healthy individuals balanced for handedness. PLoS ONE, 9 (2014), p. e101165

M. Mennes, C. Kelly, S. Colcombe, F.X. Castellanos, M.P. Milham The extrinsic and intrinsic functional architectures of the human brain are not equivalent. Cereb Cortex, 23 (2013), pp. 223-229

M. Mesulam Principles of behavioral and cognitve neurology. Oxford University Press, Oxford (2000)

M.M. Mesulam Large-scale neurocognitive networks and distributed processing for attention, language, and memory. Ann Neurol, 28 (1990), pp. 597-613

D. Meyer, E. Dimitriadou, K. Hornik, A. Weingessel, F. Leisch R package “e10701” version V1.6 (2012)

T. Minka Automatic choice of dimensionality for PCA. MIT Media Lab report 514. Report nr $514(2000)$

M. Naveau, G. Doucet, N. Delcroix, L. Petit, L. Zago, F. Crivello, et al. A novel group ICA approach based on multi-scale individual component clustering. Application to a large sample of fMRI data. Neuroinformatics, 10 (2012), pp. 269-285

R.C. Oldfield The assessment and analysis of handedness: the Edinburgh inventory.

Neuropsychologia, 9 (1971), pp. 97-113 
J. Sacher, J. Neumann, H. Okon-Singer, S. Gotowiec, A. Villringer Sexual dimorphism in the human brain: evidence from neuroimaging. Magn Reson Imaging, 31 (2013), pp. 366-375

G. Salimi-Khorshidi, G. Douaud, C.F. Beckmann, M.F. Glasser, L. Griffanti, S.M. Smith Automatic denoising of functional MRI data: combining independent component analysis and hierarchical fusion of classifiers. NeuroImage, 90 (2014), pp. 449-468

X. Shen, F. Tokoglu, X. Papademetris, R.T. Constable Groupwise whole-brain parcellation from resting-state fMRI data for network node identification. NeuroImage, 82 (2013), pp. 403-415

S.M. Smith, P.T. Fox, K.L. Miller, D.C. Glahn, P.M. Fox, C.E. Mackay, et al. Correspondence of the brain's functional architecture during activation and rest. Proc Natl Acad Sci USA, 106 (2009), pp. 13040-13045

S.M. Smith, M. Jenkinson, M.W. Woolrich, C.F. Beckmann, T.E. Behrens, H. Johansen-Berg, et al. Advances in functional and structural MR image analysis and implementation as FSL. NeuroImage, 23 (Suppl. 1) (2004), pp. S208-S219

D.E. Stark, D.S. Margulies, Z.E. Shehzad, P. Reiss, A.M. Kelly, L.Q. Uddin, et al. Regional variation in interhemispheric coordination of intrinsic hemodynamic fluctuations. J Neurosci, 28 (2008), pp. 13754-13764

Z.Y. Sun, S. Kloppel, D. Riviere, M. Perrot, R. Frackowiak, H. Siebner, et al., J.F. Mangin The effect of handedness on the shape of the central sulcus. NeuroImage, 60 (2012), pp. 332339

J. Talairach, P. Tournoux Co-planar stereotaxic atlas of the human brain 3-dimensional proportional system: an approach to cerebral imaging. Stuttgart, New York, NY (1988)

A.W. Toga, P.M. Thompson Mapping brain asymmetry. Nat Rev Neurosci, 4 (2003), pp. 3748

R. Toro, P.T. Fox, T. Paus Functional coactivation map of the human brain. Cereb Cortex, 18 (11) (2008), pp. 2553-2559

N. Tzourio-Mazoyer, M. Joliot, D. Marie, B. Mazoyer Variation in homotopic areas' activity and inter-hemispheric intrinsic connectivity with type of language lateralization: an FMRI study of covert sentence generation in 297 healthy volunteers. Brain Struct Funct (2015)

N. Tzourio-Mazoyer, B. Landeau, D. Papathanassiou, F. Crivello, O. Etard, N. Delcroix, et al. Automated anatomical labeling of activations in SPM using a macroscopic anatomical parcellation of the MNI MRI single-subject brain. NeuroImage, 15 (2002), pp. 273-289

M.P. van den Heuvel, H.E. Hulshoff Pol Specific somatotopic organization of functional connections of the primary motor network during resting state. Hum Brain Mapp, 31 (2010), pp. 631-644 
D.C. Van Essen, H.A. Drury, J. Dickson, J. Harwell, D. Hanlon, C.H. Anderson An integrated software suite for surface-based analyses of cerebral cortex. J Am Med Inform Assoc, 8 (2001), pp. 443-459

G. Varoquaux, S. Sadaghiani, P. Pinel, A. Kleinschmidt, J.B. Poline, B. Thirion A group model for stable multi-subject ICA on fMRI datasets. NeuroImage, 51 (2010), pp. 288-299

M. Vigneau, V. Beaucousin, P.Y. Herve, H. Duffau, F. Crivello, O. Houde, et al. Metaanalyzing left hemisphere language areas: phonology, semantics, and sentence processing. NeuroImage, 30 (2006), pp. 1414-1432

M. Vigneau, V. Beaucousin, P.Y. Hervé, G. Jobard, L. Petit, F. Crivello, et al. What is righthemisphere contribution to phonological, lexico-semantic, and sentence processing? Insights from a meta-analysis. NeuroImage, 54 (2010), pp. 577-593

B.T. Yeo, F.M. Krienen, J. Sepulcre, M.R. Sabuncu, D. Lashkari, M. Hollinshead, et al. The organization of the human cerebral cortex estimated by intrinsic functional connectivity. $\mathrm{J}$ Neurophysiol, 106 (2011), pp. 1125-1165

A. Zalesky, L. Cocchi, A. Fornito, M.M. Murray, E. Bullmore Connectivity differences in brain networks. NeuroImage, 60 (2012), pp. 1055-1062

K. Zilles, K. Amunts Neuroscience. Segregation and wiring in the brain. Science, 335 (2012), pp. 1582-1584

X.N. Zuo, C. Kelly, A. Di Martino, M. Mennes, D.S. Margulies, S. Bangaru, et al. Growing together and growing apart: regional and sex differences in the lifespan developmental trajectories of functional homotopy. J Neurosci, 30 (2010), pp. 15034-15043 